\title{
Involutions and linear systems on holomorphic symplectic manifolds
}

\author{
Kieran G. O'Grady* \\ Università di Roma "La Sapienza"
}

October 24,2018

\section{Introduction}

A compact Kähler manifold is irreducible symplectic if it is simply connected and it carries a holomorphic symplectic form spanning the space of global holomorphic 2-forms. A 2-dimensional irreducible symplectic manifolds is nothing else but a $K 3$ surface. The well established theory of periods of $K 3$ surfaces has been a model for the theory in higher dimensions and indeed Local Torelli [3] and Surjectivity of the period map [14, 15] hold in any dimension. K3 surfaces are remarkable not only for their periods: the complete linear system associated to an ample divisor has very simple behaviour [23] and furthermore one can describe explicitely all $K 3$ surfaces with an ample divisor whose self-intersection is small. This paper deals with the question: do similar properties hold for ample divisors on an irreducible symplectic manifold of arbitrary dimension? Deformations of $S^{[n]}$, the Hilbert scheme parametrizing length- $n$ subschemes of a $K 3$ surface $S$, are in many respects the symplest known irreducible symplectic manifolds - recall that the generic such deformation is not birational to $(K 3)^{[n]}$ (Thm. 6, p.779 of [3]) if $n \geq 2$. The L Conjecture (1.2) predicts that if $(X, H)$ is the generic couple with $X$ a deformation of $(K 3)^{[n]}$ and $H$ an ample divisor of square 2 for Beauville's quadratic form then $|H|$ has no base-locus and the map $X \rightarrow|H|^{\vee}$ has degree 2 onto its image and furthermore $c_{1}(H)$ spans the subspace of $H^{2}(X)$ fixed by the covering involution. When $n=1$ the conjecture is clearly true - every degree- 2 polarized $K 3$ is a double cover of $\mathbb{P}^{2}$. If the $\mathrm{L}$ Conjecture is true then it follows that a deformation of $(K 3)^{[n]}$ carrying a divisor (not necessarily ample) of degree 2 has an anti-symplectic birational involution: this is a non-trivial assertion, usually easier to test than the L Conjecture - we call it the I Conjecture (1.3). Before giving the precise statements we recall the properties of Beauville's quadratic form (Thm. 5, p. 772 of [3]). Let $X$ be an irreducible symplectic manifold of (complex) dimension $2 n$ : the quadratic form $(,)_{X}$ on $H^{2}(X)$ - a higher dimensional analogue of the intersection form - is characterized by the following properties:

(1) $(,)_{X}$ is integral indivisible non-degenerate, $\left(H^{p, q}, H^{p^{\prime}, q^{\prime}}\right)_{X}=0$ if $p+p^{\prime} \neq 2$.

\footnotetext{
*Supported by Cofinanziamento MURST 2002-03
} 
(2) The signature of $(,)_{X}$ is $\left(3, b_{2}(X)-3\right)$. If $H$ is ample $(,)_{X}$ is positive definite on

$$
\left(H^{2,0}(X) \oplus H^{0,2}(X)\right)_{\mathbb{R}} \oplus \mathbb{R} c_{1}(H) .
$$

(3) There is a positive rational constant $c_{X}$ such that the following formula of Fujiki holds (Thm. (4.7) of [10]):

$$
\int_{X} \alpha^{2 n}=c_{X} \cdot(\alpha, \alpha)_{X}^{n}
$$

Both $c_{X}$ and $(,)_{X}$ do not change if we modify the complex structure of $X$. Beauville's form and the Fujiki constant of $(K 3)^{[n]}$ are given in Subsubsection (4.1.1). Huybrechts (Lemma (2.6) of [14) proved that $(,)_{X}$ behaves well with respect to birational maps. More precisely let $\phi: X \cdots>Y$ be a birational (i.e. bimermomorphic) map between irreducible symplectic manifolds and $H^{2}(\phi): H^{2}(Y ; \mathbb{Z}) \rightarrow H^{2}(X ; \mathbb{Z})$ be defined by the Künneth decomposition of the Poincaré dual of the graph of $\phi$. Then $H^{2}(\phi)$ is an isometry of lattices. Furthermore, letting $\operatorname{Bir}(X)$ be the group of birational maps of $X$ to itself, the map

$$
\begin{array}{ccc}
\operatorname{Bir}(X) & \stackrel{H^{2}}{\longrightarrow} & \operatorname{Isom}\left(H^{2}(X ; \mathbb{Z}),(,)_{X}\right) \\
\phi & \mapsto & H^{2}(\phi)
\end{array}
$$

is a homomorphism into the subgroup of integral Hodge isometries of $H^{2}(X ; \mathbb{C})$. Whenever no confusion may arise we denote $(,)_{X}$ by $($,$) . For h \in H_{\mathbb{Z}}^{1,1}(X)$ with $(h, h)=2$ we let $R_{h}: H^{2}(X) \rightarrow H^{2}(X)$ be the reflection in the span of $h$, i.e.

$$
R_{h}(v)=-v+(v, h) h .
$$

Definition 1.1. A couple $(X, H)$ is a degree- $k$ polarized irreducible symplectic variety if $X$ is an irreducible symplectic manifold and $H$ is an indivisible ample divisor on $X$ with $\left(c_{1}(H), c_{1}(H)\right)=k$.

Now let $(X, H)$ be a degree-2 polarized irreducible symplectic variety with $X$ a deformation of $(K 3)^{[n]}$. By a theorem of Kollár-Matsusaka 19 there is an $\ell_{n}>0$ depending only on $n$ such that the a priori rational map $X \cdots>\left|\ell_{n} H\right|^{\vee}$ is in fact a regular embedding. Thus all $(X, H)$ as above are realizable as subvarieties of a $\mathbb{P}^{d_{n}}$ with a fixed Hilbert polynomial $p_{n}$ : let $\mathcal{Q}_{n}$ be the Hilbert scheme to which they "belong" and let $\mathcal{Q}_{n}^{0} \beta \mathcal{Q}_{n}$ be the open subset given by

$$
\mathcal{Q}_{n}^{0}:=\left\{t \in \mathcal{Q}_{n} \mid X_{t} \text { is irreducible symplecic }\right\},
$$

where $X_{t}$ is the subvariety of $\mathbb{P}^{d_{n}}$ corresponding to $t \in \mathcal{Q}_{n}$.

Conjecture 1.2. [L Conjecture] Keep notation as above. There exists an open dense subset $U_{n} ß \mathcal{Q}_{n}^{0}$ such that for $t \in U_{n}$ the following holds. Let $\left(X_{t}, H_{t}\right)$ be the degree-2 polarized irreducible symplectic variety corresponding to $t$. Then $\left|H_{t}\right|$ has no base-locus and $f_{t}: X_{t} \rightarrow\left|H_{t}\right|^{\vee}$ is of degree 2 onto its image $Y_{t}$. In particular there exists an involution $\phi_{t}: X_{t} \rightarrow X_{t}$ such that $f_{t}$ is the composition

$$
X_{t} \stackrel{\pi_{t}}{\longrightarrow} X_{t} /\left\langle\phi_{t}\right\rangle \stackrel{\nu_{t}}{\longrightarrow} Y_{t}
$$

where $\pi_{t}$ is the quotient map and $\nu_{t}$ is the normalization map. Let $h_{t}:=c_{1}\left(H_{t}\right)$. Then

$$
H^{2}\left(\phi_{t}\right)=R_{h_{t}} .
$$


In the next section we will show that if the above conjecture holds then also the following conjecture is true.

Conjecture 1.3. [I Conjecture] Let $X$ be an irreducible symplectic manifold deformation equivalent to $(K 3)^{[n]}$. Suppose that $h \in H_{\mathbb{Z}}^{1,1}(X)$ and $(h, h)=2$. There exists a birational involution $\phi: X \cdots>X$ such that for $\gamma \in H^{2}(X)$

$$
H^{2}(\phi)(\gamma)=R_{h}(\gamma)-\sum_{i}\left(\gamma, \alpha_{i}\right) \beta_{i}
$$

where $\alpha_{i} \in H_{\mathbb{Q}}^{1,1}(X)$ with $\left(\alpha_{i}, \cdot\right)$ equal to integration over an effective analytic 1-cycle and $\beta_{i} \in H_{\mathbb{Z}}^{1,1}(X)$ is Poincaré dual to an effective divisor.

A few comments. It follows from (1.0.6) that $H^{2}(\phi)$ multiplies a symplectic form by $(-1)$ : in particular $\phi$ is not the identity! Since (1.0.2) is a homomorphism $H^{2}(\phi)$ is an involution: this imposes restrictions on the $\alpha_{i}$ 's and $\beta_{i}$ 's.

After proving that the L Conjecture implies the I Conjecture we will show this is easy - that the two conjectures are stable under deformations if certain hypotheses are satisfied. More precisely: If $X, h, \phi$ are as in the I Conjecture and furthermore $\phi$ is regular with $H^{2}(\phi)=R_{h}$ then $\phi$ extends to all small deformations of $X$ that keep $h$ of type $(1,1)$. If we have $t_{0} \in \mathcal{Q}_{n}^{0}$ such that $\left(X_{t_{0}}, H_{t_{0}}\right)$ behaves as stated in the $\mathrm{L}$ Conjecture then the same holds for $\left(X_{t}, H_{t}\right)$ where $t$ varies in an open subset of $\mathcal{Q}_{n}^{0}$ containing (the orbit of) $\left(X_{t_{0}}, H_{t_{0}}\right)$. In the next section we give examples of couples $X, h, \phi$ where $X$ is a deformation of $(K 3)^{[n]}, h \in H_{\mathbb{Z}}^{1,1}(X)$ has degree 2 for Beauville's form and $\phi$ is a rational involution of $X$ with $H^{2}(\phi)=R_{h}$. In our examples $X$ is always a moduli space of rank- $r$ torsion-free sheaves on a $K 3$ surface $S$. The involutions were introduced by Beauville [4] in the rank-1 case, by Mukai [26] when $r \geq 2$. The new example in Subsubsection (4.3) is a generalized Mukai reflection. We spend some time proving that the action on $H^{2}$ is indeed the reflection in a class $h$ of square 2: once this is proved we know that the regular involutions are stable under small deformations of $(X, h)$. There are examples of regular involutions in any (even) dimension as long as we allow $r$ to be arbitrarily large. We expect that all of the examples we give (with the possible exception of the one in Subsubsection (4.3) are "polarized" deformation equivalent but we do not prove this, see Section ([6). One should notice that if we have 2 regular involutions $\phi_{1}, \phi_{2}$ on the same $X$ with $H^{2}\left(\phi_{i}\right)=R_{h_{i}}$ where $h_{1}, h_{2}$ are independent then $\phi_{1} \circ \phi_{2}$ is an automorphism of infinite order generating an interesting dynamical system. If furthermore $X$ and $\phi_{1}, \phi_{2}$ are defined over a number field $K$ one may study the action of $\phi_{1} \circ \phi_{2}$ on $X(\bar{K})$ : this was done by Silverman [31] for $X$ a $K 3$. We briefly discuss this in Subsection (4.4). Section (5D) is devoted to examples of degree-2 polarized $(X, H)$ where $X$ is a deformation of $(K 3)^{[n]}$ and $|H|$ has the good behaviour stated in the L Conjecture. We give examples in dimensions 4,6,8. In doing so we prove that the so-called Strange duality statement [8, 20, 7, 21] holds for certain couples of moduli spaces of sheaves on a $K 3$. We examine more closely the 4-dimensional example (first given by Mukai 29]): a moduli space $X$ of rank2 sheaves on a $K 3$ surface $S \beta \mathbb{P}^{6}$ of degree-10, with $H$ a suitable ample divisor. We have an identification $|H|^{\vee} \cong\left|I_{S}(2)\right|$ (Strange duality) and the image of $X \rightarrow\left|I_{S}(2)\right|$ is the non-degenerate component, call it $Y$, of the hypersurface parametrizing singular quadrics, the other component being a hyperplane. $Y$ is a sextic 4 -fold in $\mathbb{P}^{5}$, singular along a smooth surface; Conjecture L predicts 
that the generic degree-2 polarized $(X, H)$ with $X$ a deformation of $(K 3)^{[2]}$ is a double cover of a sextic 4 -fold in $\mathbb{P}^{5}$. This explains one of the main motivations for formulating our conjectures. If the $\mathrm{L}$ Conjecture is true in dimension 4 then we should have a relatively explicit way of describing all degree-2 polarized $(X, H)$ with $X$ a deformation of $(K 3)^{[2]}$ and hence also the relevant moduli space, call it $M_{2}$. In this respect we notice that all known explicit constructions of irreducible projective symplectic varieties give families of codimension 1 in the relevant moduli space, with one exception - the variety of lines on a cubic 4-fold [5]: in this case we get a whole component of the moduli space $M_{6}$ of degree-6 polarized $(X, H)$ with $X$ a deformation of $(K 3)^{[2]}$ and $\left(c_{1}(H), \cdot\right)$ a functional on $H^{2}(X ; \mathbb{Z})$ divisible by 2 . If $M_{6}$ is irreducible then global Torelli for deformations of $(K 3)^{[2]}$ follows from Voisin's Torelli Theorem [32] for cubic 4 -folds. We do not know how to attack the problem of irreducibily of $M_{6}$; on the other hand the L Conjecture should allow us to describe $M_{2}$, and once this is done we should be in a better position to study the period map. Going back to Mukai's example we notice that the dual hypersurface $Y^{\vee} \beta\left(\mathbb{P}^{5}\right)^{\vee}$ is the image of another symplectic variety $X^{\vee}$, in fact $X^{\vee}=S^{[2]}$, via the complete linear system associated to a certain degree-2 divisor $H^{\vee}$ which is base-point free but not ample - it contracts a $\mathbb{P}^{2}$. We expect that there is an involution on the moduli space of degree-2 quasi-polarized $(X, H)$ ( $X$ a deformation of $\left.(K 3)^{[2]}\right)$ which generalizes the above duality: we will give some evidence for this in a forthcoming paper on the L Conjecture in dimension 4.

\section{The L Conjecture implies the I Conjecture}

Set $X_{0}=X$ and $h_{0}=h$. By Bogomolov's Theorem $[6] X_{0}$ has a smooth versal deformation space and hence there exists a proper submersive map $\pi: \mathcal{X} \rightarrow B$ between manifolds and a point $0 \in B$ with the following properties: $\pi^{-1}(0) \cong X_{0}$ and the Kodaira-Spencer map

$$
T_{B, 0} \rightarrow H^{1}\left(T_{X_{0}}\right)
$$

is an isomorphism. The germ $\left(\mathcal{X}, X_{0}\right) \rightarrow(B, 0)$ is identified with the deformation space of $X_{0}$ : we say that $\pi$ is a representative of the versal deformation space of $X_{0}$. We set $X_{t}:=\pi^{-1}(t)$. The holomorphic symplectic form on $X_{0}$ defines an isomorphism $T_{X_{0}} \cong \varnothing_{X_{0}}^{1}$ and hence $H^{1}\left(T_{X_{0}}\right) \cong H^{1}\left(\varnothing_{X_{0}}^{1}\right)$ : since $h^{1}\left(\varnothing_{X_{0}}^{1}\right)=21$ (see Prop. 6, p. 768 of [3]) we get by (2.0.1) that

$$
\operatorname{dim} B=21 .
$$

We assume that $B$ is simply connected and therefore there is a well-defined period map

$$
\begin{array}{ccc}
B & \stackrel{P}{\longrightarrow} & \mathbb{P}\left(H^{2}\left(X_{0}\right)\right) \\
t & \mapsto & g_{t}^{-1}\left(H^{2,0}\left(X_{t}\right)\right)
\end{array}
$$

where $g_{t}: H^{2}\left(X_{0}\right) \rightarrow H^{2}\left(X_{t}\right)$ is given by parallel transport with respect to the Gauss-Manin connection (see for example (9.2) of 34]) along any path from 0 to $t$. As is well-known (see Thm (7.3), p. 254 of [2]) $P$ defines an isomorphism of a neighborhood of 0 onto an open subset (in the classical topology) of the smooth quadric

$$
Q:=\left\{\ell \in \mathbb{P}\left(H^{2}\left(X_{0}\right)\right) \mid(\ell, \ell)=0\right\} .
$$


Let $B\left(h_{0}\right) \beta B$ be the subset of $t$ such that $g_{t}\left(h_{0}\right) \in H^{2}\left(X_{t}\right)$ is of type $(1,1)$ : thus $B\left(h_{0}\right)=P^{-1}\left(h_{0}^{\perp}\right)$. Since $P$ is a local isomorphism

$$
B\left(h_{0}\right) \text { is smooth of codimension } 1 \text { near } 0 \text {; }
$$

by (2.0.2) we get that $\operatorname{dim} B\left(h_{0}\right)=20$. Let

$$
B\left(h_{0}\right)_{a m}:=\left\{t \in B\left(h_{0}\right) \mid h_{t} \text { or }-h_{t} \text { is the class of an ample divisor }\right\} .
$$

This is a Zariski-open subset of $B\left(h_{0}\right)$; we claim that

$$
B\left(h_{0}\right)_{a m} \neq \emptyset .
$$

In fact by the stated property of the period map $P$ the set

$$
B\left(h_{0}\right)_{1}:=\left\{t \in B\left(h_{0}\right) \mid H_{\mathbb{Z}}^{1,1}\left(X_{t}\right)=\mathbb{Z} h_{t}\right\}
$$

is the complement of a countable union of proper hypersurfaces of $B\left(h_{0}\right)$, hence there exists $\bar{t} \in B\left(h_{0}\right)_{1}$; by Huybrechts' Projectivity Criterion [14, 15] $\bar{t} \in$ $B\left(h_{0}\right)_{a m}$. Changing sign to $h_{0}$ if necessary we can assume that $h_{t}$ is ample for $t \in B\left(h_{0}\right)_{a m}$. Now assume the L Conjecture (1.2): then there is a Zariski open non-empty subset

$$
B\left(h_{0}\right)_{g d} ß B\left(h_{0}\right)_{a m}
$$

such that for $t \in B\left(h_{0}\right)_{g d}$ the complete linear system $\left|H_{t}\right|$ enjoys the properties stated in (1.2). (Here $H_{t} \in \operatorname{Pic}\left(X_{t}\right)$ is the divisor class such that $c_{1}\left(H_{t}\right)=h_{t}$.) Let $\phi_{t}: X_{t} \rightarrow X_{t}$ be the covering involution and $\Gamma_{t} \beta X_{t} \times X_{t}$ be the graph of $\phi_{t}$. We prove the I Conjecture 1.3 by considering the limit of $\Gamma_{t}$ as $t \rightarrow 0$. We view $\Gamma_{t}$ as an element of the space $\mathcal{C}_{2 n}\left(\mathcal{X} \times_{B} \mathcal{X}\right)$ parametrizing effective compactly supported analytic $2 n$-cycles constructed by Barlet [1. Let $\left\{t_{k}\right\}_{k \in \mathbb{N}}$ be a sequence of points $t_{k} \in B\left(h_{0}\right)_{g d}$ converging to 0 ; such a sequence exists because $B\left(h_{0}\right)_{g d}$ is a Zariski-open and dense subset of $B\left(h_{0}\right)$. Proceeding exactly as in the proof of Theorem (4.3) of [14] we see that passing to a subsequence we can assume that $\left\{\Gamma_{t_{k}}\right\}_{k \in \mathbb{N}}$ converges to an effective analytic $2 n$-cycle $\Gamma_{0}$ on $X_{0} \times X_{0}$. This (see the proof of Theorem (4.3) of [14]) implies that there is a decomposition

$$
\Gamma_{0}=\Gamma\left(\phi_{0}\right)+\sum_{i} n_{i} \varnothing_{i}
$$

where $\phi_{0}: X_{0} \cdots>X_{0}$ is a birational map, $\Gamma\left(\phi_{0}\right)$ is the graph of $\phi_{0}, n_{i}>0, \varnothing_{i}$ is irreducible and $\varnothing_{i} \beta D_{i} \times E_{i}$ where $D_{i}, E_{i} \beta X_{0}$ are proper subsets. Since $\Gamma_{t_{k}}$ is invariant for the involution of $X_{t_{k}} \times X_{t_{k}}$ interchanging the factors the same must hold for $\Gamma_{0}$; this implies that $\Gamma\left(\phi_{0}\right)$ is invariant for the involution, i.e. $\phi_{0}$ is a birational involution. Finally let's show that $H^{2}\left(\phi_{0}\right)$ is as stated in the I Conjecture (1.3). For $\mathrm{E}$ an analytic cycle on $X_{0} \times X_{0}$ we let $H^{2}(\mathrm{E}): H^{2}\left(X_{0}\right) \rightarrow$ $H^{2}\left(X_{0}\right)$ be the map defined by the Künneth component in $H^{2}\left(X_{0}\right) \otimes H^{2 n-2}\left(X_{0}\right)$ of the Poincairé dual of $\mathrm{E}$. Let $G_{t}: H^{*}\left(X_{0} \times X_{0}\right) \rightarrow H^{*}\left(X_{t} \times X_{t}\right)$ be given by Gauss-Manin parallel transport along any path going from 0 to $t$ : since $G_{t}\left(\left[\Gamma_{0}\right]\right)=\left[\Gamma_{t_{k}}\right]$ we have

$$
H^{2}\left(\Gamma_{0}\right)=G_{t}^{-1} H^{2}\left(\Gamma_{t_{k}}\right)=G_{t}^{-1} H^{2}\left(\phi_{t_{k}}\right)=G_{t}^{-1} R_{h_{t}}=R_{g_{t}^{-1} h_{t}}=R_{h_{0}},
$$

where the third equality follows from 1.0.5). Now we determine $H^{2}\left(\varnothing_{i}\right)$. If $\operatorname{cod}\left(D_{i}, X\right)>1$ then $H^{2}\left(\varnothing_{i}\right)=0$. Assume that $\operatorname{cod}\left(D_{i}, X\right)=1$ and let $C_{i}$ be a 
generic fiber of the map $\emptyset_{i} \rightarrow D_{i}$ induced by the projection $X_{0} \times X_{0} \rightarrow X_{0}$ to the first factor; thus $C_{i}$ is a curve. Then

$$
H^{2}\left(\varnothing_{i}\right)(\gamma)=\left(\int_{p_{*} C_{i}} \gamma\right)\left[D_{i}\right]
$$

where $p: X_{0} \times X_{0} \rightarrow X_{0}$ is the projection to the second factor. This equation together with (2.0.5) proves that (1.0.6) holds.

\section{Stability results}

Let $X_{0}$ be an irreducible symplectic manifold - not necessarily a deformation of $(K 3)^{[n]}$. A (regular) involution $\phi_{0}: X_{0} \rightarrow X_{0}$ is anti-symplectic if

$$
\phi_{0}^{*} \sigma_{0}=-\sigma_{0}
$$

where $\sigma_{0}$ is a holomorphic symplectic form on $X$. We have an orthogonal decomposition into eigenspaces

$$
H^{2}\left(X_{0} ; \mathbb{Q}\right)=H^{2}\left(\phi_{0}\right)(+1)_{\mathbb{Q}} \oplus_{\perp} H^{2}\left(\phi_{0}\right)(-1)_{\mathbb{Q}} .
$$

\subsection{Involutions}

Assume $X_{0}$ and $\phi_{0}$ are as above. By Bogomolov's Theorem [6] $X_{0}$ has a smooth versal deformation space; let $\pi: \mathcal{X} \rightarrow B$ be a representative for the deformation space of $X_{0}$ and $X_{t}:=\pi^{-1}(t)$. Let

$$
B\left(\phi_{0}\right):=\left\{t \in B \mid \exists \phi_{t}: X_{t} \rightarrow X_{t} \text { deformation of } \phi_{0}\right\} .
$$

Equation (3.0.1) gives that $H^{2,0}\left(X_{0}\right) B H^{2}\left(\phi_{0}\right)(-1)$ and hence

$$
L_{0}:=H^{2}\left(\phi_{0}\right)(+1)_{\mathbb{Q}} B H_{\mathbb{Q}}^{1,1}\left(X_{0}\right) .
$$

Let

$$
B\left(L_{0}\right):=\left\{t \in B \mid g_{t}\left(L_{0}\right) ß H_{\mathbb{Q}}^{1,1}\left(X_{t}\right)\right\}
$$

where $g_{t}: H^{2}\left(X_{0}\right) \rightarrow H^{2}\left(X_{t}\right)$ is given by Gauss-Manin parallel transport along any path connecting 0 to $t$ (we assume that $B$ is simply connected).

Proposition 3.1. Keep notation and assumptions as above. In a neighborhood of 0 we have $B\left(\phi_{0}\right)=B\left(L_{0}\right)$.

Proof. By the universal property of the deformation space there exist an involution $\tau: B \rightarrow B$ fixing 0 and an isomorphism $\Phi: \tau^{*} \mathcal{X} \rightarrow \mathcal{X}$ of families over $B$ whose restriction to $X_{0}$ is equal to $\phi_{0}$. (We allow ourselves to shrink $B$.) Let $B^{\tau} \beta B$ be the fixed locus of $\tau$; then

$$
B^{\tau} \beta B\left(\phi_{0}\right) \beta B\left(L_{0}\right) .
$$

Since $B^{\tau}$ is smooth it suffices to prove that

$$
T_{B^{\tau}, 0}=T_{B\left(L_{0}\right), 0} .
$$


Let $\omega_{0}$ be a symplectic form on $X_{0}$; contraction with $\omega_{0}$ defines an isomorphism

$$
T_{B, 0}=H^{1}\left(T_{X_{0}}\right) \stackrel{\sim}{\longrightarrow} H^{1}\left(\varnothing_{X_{0}}^{1}\right) .
$$

With this identification we have $T_{B\left(L_{0}\right), 0}=L_{0}^{\perp}=H^{2}\left(\phi_{0}\right)(-1)$. On the other hand Isomorphism (3.1.3) gives an identification

$$
T_{B^{\tau}, 0}=H^{2}\left(\phi_{0}\right)(-1)
$$

because $\phi_{0}$ is anti-symplectic. This proves (3.1.2).

As an immediate consequence we have the following result.

Corollary 3.2. Keep notation and hypotheses as above. Assume furthermore that $H^{2}\left(\phi_{0}\right)=R_{h_{0}}$ where $h_{0} \in H_{\mathbb{Z}}^{1,1}\left(X_{0}\right)$. Then $\phi_{0}$ extends to all small deformations of $X_{0}$ that keep $h_{0}$ of type $(1,1)$.

\subsection{Linear systems}

Let $\left(X_{0}, H_{0}\right)$ be a degree-2 polarized deformation of $(K 3)^{[n]}$. Let $\mathcal{Q}_{n}^{0}$ be the open subset of a Hilbert scheme given by (1.0.4); thus we may think that $0 \in \mathcal{Q}_{n}^{0}$ and that $X_{0}$ is the (embedded) variety corresponding to 0 , with $\mathcal{O}_{X_{0}}(1) \cong$ $\mathcal{O}_{X_{0}}\left(\ell_{n} H_{0}\right)$. Notice that $\mathcal{Q}_{n}^{0}$ is smooth at 0 because by (2.0.3) the deformation space of $\left(X_{0}, H_{0}\right)$ is smooth: let $\mathcal{A} ß \mathcal{Q}_{n}^{0}$ be the irreducible component containing 0 .

Proposition 3.3. Keep notation as above. Suppose that $\left|H_{0}\right|$ has no base-locus and that $f_{0}: X_{0} \rightarrow\left|H_{0}\right|^{\vee}$ is of degree 2 onto its image $Y_{0}$, hence there exists an involution $\phi_{0}: X_{0} \rightarrow X_{0}$ such that $f_{0}$ is the composition

$$
X_{0} \stackrel{\pi_{0}}{\longrightarrow} X_{0} /\left\langle\phi_{0}\right\rangle \stackrel{\nu_{0}}{\longrightarrow} Y_{0}
$$

where $\pi_{0}$ is the quotient map and $\nu_{0}$ is the normalization map. Suppose also that

$$
H^{2}\left(\phi_{0}\right)=R_{h_{0}}
$$

where $h_{0}:=c_{1}\left(H_{0}\right)$. Then there exists an open non-empty subset $\mathcal{V} B \mathcal{A}$ such that the statements above hold when we replace $\left(X_{0}, H_{0}\right)$ by $\left(X_{t}, H_{t}\right)$.

Proof. Having no base-locus is an open property; since $\left|H_{0}\right|$ has no base-locus we get that $\left|H_{t}\right|$ has have no base-locus for $t$ varying in an open non-empty subset of $\mathcal{A}$. Now consider the other statements. First locally around 0 we can extract the $\ell_{n}$-th root of $\mathcal{O}_{X_{t}}(1)$. More precisely there exist a quasi-projective manifold $\mathcal{U}$, a finite map $\rho: \mathcal{U} \rightarrow \mathcal{A}$ and a point $\widetilde{0} \in \mathcal{U}$ with the following properties: $\rho(\widetilde{0})=0$, $\rho$ is submersive at $\widetilde{0}$ and the pull-back $\zeta: \mathcal{X} \rightarrow \mathcal{U}$ of the tautological family over $\mathcal{A}$ carries a divisor class $\mathcal{H}$ such that for $t \in U$ we have $\left(c_{1}\left(H_{t}\right), c_{1}\left(H_{t}\right)\right)=2$ and $\mathcal{O}_{X_{t}}(1) \cong \mathcal{O}_{X_{t}}\left(\ell_{n} H_{t}\right)$. (Here $X_{t}:=\pi^{-1}(t)$ and $H_{t}:=\left.\mathcal{H}\right|_{X_{t}}$.) By Corollary (3.2) there exists a Zariski-open $\mathcal{U}_{i n v} ß \mathcal{U}$ such that for $t \in \mathcal{U}_{i n v}$ we have an involution $\phi_{t}: X_{t} \rightarrow X_{t}$ with $H^{2}\left(\phi_{t}\right)=R_{h_{t}}$. Let $\mathcal{X}_{i n v}:=\pi^{-1}\left(\mathcal{U}_{i n v}\right)$; then we have an involution $\Phi: \mathcal{X}_{i n v} \rightarrow \mathcal{X}_{i n v}$ restricting to $\phi_{t}$ on each $X_{t}$. Let $\widetilde{\mathcal{Y}}:=\mathcal{X}_{i n v} /\langle\Phi\rangle$ be the quotient. The map $\xi: \widetilde{\mathcal{Y}} \rightarrow \mathcal{U}_{i n v}$ is analytically locally trivial hence $\xi$ is a flat family. Let $\mathcal{H}_{i n v}$ be the restriction of $\mathcal{H}$ to $\mathcal{X}_{\text {inv }}$. We claim that $\mathcal{H}_{\text {inv }}$ descends to a divisor class $\overline{\mathcal{H}}$ on $\widetilde{\mathcal{Y}}$ : this follows at once from the fact that by 
hypothesis $H_{0}$ descends to the divisor class on $\widetilde{Y}_{0}$ given by $\nu_{0}^{*} \mathcal{O}_{Y_{0}}(1)$. Letting $f_{t}: X_{t} \rightarrow \widetilde{Y}_{t}$ be the quotient map we have the pull-back

$$
f_{t}^{*}: H^{0}\left(\tilde{Y}_{t} ; \bar{H}_{t}\right) \rightarrow H^{0}\left(X_{t} ; H_{t}\right) .
$$

We claim that $f_{t}^{*}$ is an isomorphism for all $t \in \mathcal{U}_{i n v}$. Since $f_{t}^{*}$ is injective it suffices to check that

$$
h^{0}\left(\widetilde{Y}_{t} ; \bar{H}_{t}\right)=h^{0}\left(X_{t} ; H_{t}\right) .
$$

We claim that

$$
h^{p}\left(\widetilde{Y}_{t} ; \bar{H}_{t}\right)=0=h^{p}\left(X_{t} ; H_{t}\right), \quad p>0 .
$$

This follows from the classical Kodaira vanishing for $X_{t}$ because $K_{X_{t}} \sim 0$ and for $\widetilde{Y}_{t}$ we apply for example Theorem (1-2-5) of [18; notice that $\widetilde{Y}_{t}$ has terminal singularities and $K_{\widetilde{Y}_{t}} \equiv 0$ (for this we need $\operatorname{dim} X_{0} \geq 4$, if $\operatorname{dim} X_{0}=2$ we are considering $K 3$ surfaces and the proposition is trivially verified). From (3.2.4) we get that in order to prove (3.2.3) it suffices to show that $\chi\left(\widetilde{Y}_{t} ; \bar{H}_{t}\right)=\chi\left(X_{t} ; H_{t}\right)$. Since $\mathcal{X}_{i n v} \rightarrow \mathcal{U}_{i n v}$ and $\widetilde{\mathcal{Y}} \rightarrow \mathcal{U}_{i n v}$ are flat families and $\mathcal{U}_{i n v}$ is connected it is enough to check that $\chi\left(\widetilde{Y}_{0} ; \bar{H}_{0}\right)=\chi\left(X_{0} ; H_{0}\right)$. By (3.2.4) this is equivalent to $h^{0}\left(\widetilde{Y}_{0} ; \bar{H}_{0}\right)=h^{0}\left(X_{0} ; H_{0}\right)$ : this we know by hypothesis. We have proved that (3.2.2) is an isomorphism. It follows that there is an open non-empty $\mathcal{U}_{i n v}^{\prime} \Re_{i n v}$ such that for $t \in \mathcal{U}_{i n v}^{\prime}$ the map $X_{t} \rightarrow Y_{t} ß\left|H_{t}\right|^{\vee}$ factors through the quotient map $X_{t} \rightarrow \widetilde{Y}_{t}$ and that the induced map $\widetilde{Y}_{t} \rightarrow Y_{t}$ is the normalization map. Finally $H^{2}\left(\phi_{t}\right)$ is constant with respect to the Gauss-manin connection because $\phi_{t}$ is regular for all $t \in \mathcal{U}_{i n v}^{\prime}$ and hence $H^{2}\left(\phi_{t}\right)=R_{h_{t}}$ because (3.2.1) holds.

\section{Examples: involutions}

We give examples of birational involutions on deformations of $(K 3)^{[n]}$ whose action on $H^{2}$ is the reflection in a $(1,1)$-class $h$. In many cases the involution is regular and hence by Corollary (3.2) it will extend to all small deformations keeping $h$ of type $(1,1)$.

\subsection{Beauville's examples}

These are involutions of $S^{[n]}$ where $S$ is a $K 3$ surface; they were introduced by Beauville, see pp. 20-25 of [4].

\subsubsection{Hilbert scheme of points on a $K 3$}

We recall the description of $H^{2}\left(S^{[n]}\right)$ and its Beauville form (see Prop. 6, p.768 of [3], the remark following it and pp. 777-778). Let $S^{(n)}$ be the symmetric product of $n$ copies of $S$; we have a natural "symmetrization" map $s: H^{2}(S ; \mathbb{Z}) \rightarrow$ $H^{2}\left(S^{(n)} ; \mathbb{Z}\right)$. The cycle map $c: S^{[n]} \rightarrow S^{(n)}$ gives rise to $c^{*}: H^{2}\left(S^{(n)} ; \mathbb{Z}\right) \rightarrow$ $H^{2}\left(S^{[n]}\right)$. Composing $s$ and $c^{*}$ we get the map

$$
\mu: H^{2}(S ; \mathbb{Z}) \rightarrow H^{2}\left(S^{[n]} ; \mathbb{Z}\right)
$$

which is an injection onto a saturated subgroup of $H^{2}\left(S^{[n]} ; \mathbb{Z}\right)$. Let

$$
\Delta_{n}:=\left\{[Z] \in S^{[n]} \mid Z \text { is non-reduced }\right\},
$$


i.e. the exceptional divisor of $c$ : there exists a (unique) divisor-class $\Xi_{n}$ such that $2 \Xi_{n} \sim \Delta_{n}$, set $\xi_{n}:=c_{1}\left(\Xi_{n}\right)$. There is a direct sum decomposition

$$
H^{2}\left(S^{[n]} ; \mathbb{Z}\right)=\mu\left(H^{2}(S ; \mathbb{Z})\right) \oplus_{\perp} \mathbb{Z} \xi_{n}
$$

orthogonal with respect to Beauville's form, and furthermore

$$
\begin{aligned}
(\mu(\alpha), \mu(\beta)) & =\int_{S} \alpha \wedge \beta \\
\left(\xi_{n}, \xi_{n}\right) & =-2(n-1) .
\end{aligned}
$$

Since $\mu$ is a morphism of Hodge structures and $\xi_{n}$ is of type $(1,1)$ Equality 4.1.3) determines the Hodge structure of $H^{2}\left(S^{[n]}\right)$. Finally we recall that the Fujiki constant (see (1.0.1) ) of $X=S^{[n]}$ is given by

$$
c_{X}=\frac{(2 n) !}{n ! 2^{n}} .
$$

\subsubsection{The involution}

Assume that $D_{2 g-2}$ is a globally generated ample divisor on $S$ with

$$
D_{2 g-2} \cdot D_{2 g-2}=2 g-2 .
$$

If $[Z] \in S^{[g-1]}$ is generic then $\left|I_{Z}\left(D_{2 g-2}\right)\right|$ is one-dimensional and its base-locus (as a linear system of divisors on $S$ ) equals $Z \amalg W$, where $W$ is a length- $(g-1)$ subscheme of $S$ : Beauville defines a birational involution

$$
\phi: S^{[g-1]} \cdots>S^{[g-1]}
$$

by setting $\phi([Z]):=[W]$ for the generic $[Z] \in S^{[g-1]}$. For $g=2$ the map $\phi$ is the involution defined on a $K 3$ of degree-2. For $g \geq 3$ and $D_{2 g-2}$ very ample we have $S \beta \mathbb{P}^{g}$ and for $[Z]$ generic $Z \coprod W=\langle Z\rangle \cap S$ where $\langle Z\rangle$ is the $(g-2)$-dimensional span of $Z$. We will study $H^{2}(\phi)$. Let

$$
h_{g}:=\left(\mu\left(c_{1}\left(D_{2 g-2}\right)\right)-\xi_{g-1}\right) \in H_{\mathbb{Z}}^{1,1}\left(S^{[g-1]}\right) .
$$

Notice that $\left(h_{g}, h_{g}\right)=2$.

Proposition 4.1. For $\left(S, D_{2 g-2}\right)$ varying in an open dense subset of the relevant moduli space of polarized K3 surfaces $H^{2}(\phi)$ equals the reflection in the span of $h_{g}$ i.e.

$$
H^{2}(\phi)(\gamma)=R_{h_{g}}:=-\gamma+\left(\gamma, h_{g}\right) h_{g} .
$$

Remark 4.2. The "relevant moduli space..." means the following. Let $D_{2 g-2} \sim$ $k D_{2 \bar{g}-2}$ where $k \in \mathbb{N}$ and $c_{1}\left(D_{2 \bar{g}-2}\right)$ is indivisible: the relevant moduli space is that of degree- $(2 \bar{g}-2)$ polarized $K 3$ 's.

Proof of Proposition (4.1). First consider the case $g=2$ : then $S /\langle\phi\rangle \cong \mathbb{P}^{2}$ hence the $(+1)$-eigenspace of $H^{2}(\phi)$ is generated by $h_{2}$ and thus $H^{2}(\phi)$ is the reflection in the span of $h_{2}$. Now assume that $g \geq 3$. By general considerations there is an open dense subset $U$ of the moduli space such that $H^{2}(\phi)$ is "constant" over $U$. Shrinking $U$ if necessary we can assume that $D_{2 g-2}$ is very ample for all $\left[\left(S, D_{2 g-2}\right)\right] \in U$. For $\left[\left(S, D_{2 g-2}\right)\right] \in U$ let

$$
f: S^{[g-1]} \cdots>\mathbf{G r}\left(1,\left|D_{2 g-2}\right|\right)
$$


be the rational map sending $[Z]$ to $\left|I_{Z}\left(D_{2 g-2}\right)\right|$, and let $L$ be the (very ample) line bundle on $\operatorname{Gr}\left(1,\left|D_{2 g-2}\right|\right)$ defined by the Plücker embedding. Since $\phi$ commutes with $f$ we have

$$
\phi^{*} f^{*} c_{1}(L)=f^{*} c_{1}(L) .
$$

We claim that

$$
f^{*} c_{1}(L)=h_{g} .
$$

It suffices to prove (4.1.9) for one $\left(S, D_{2 g-2}\right) \in U$ because $U$ is irreducible. By Hodge theory there exists $\left(S, D_{2 g-2}\right) \in U$ such that

$$
H_{\mathbb{Q}}^{1,1}(S)=\mathbb{Q} c_{1}\left(D_{2 g-2}\right) .
$$

By 4.1.3) we have

$$
H_{\mathbb{Q}}^{1,1}\left(S^{[g-1]}\right)=\mathbb{Q} \mu\left(c_{1}\left(D_{2 g-2}\right)\right) \oplus \mathbb{Q} \xi_{g-1} .
$$

Thus $f^{*} c_{1}(L)=x \mu\left(c_{1}\left(D_{2 g-2}\right)\right)+y \xi_{g-1}$. We get $x=1$ and $y=-1$ by intersecting with the algebraic 1-cycles

$$
\begin{aligned}
& \Gamma:=\left\{\left[p_{1}+\cdots p_{g-2}+p\right] \mid p \in C\right\}, \\
& \mathrm{E}:=\left\{\left[p_{1}+\cdots p_{g-3}+Z^{\prime}\right] \mid Z^{\prime} \text { non-reduced }\right\},
\end{aligned}
$$

where $p_{1}, \ldots, p_{g-2}$ are fixed and $C \in\left|D_{2 g-2}\right|$; one must recall that

$$
\left\langle c_{1}\left(\Delta_{g-1}\right), \mathrm{E}\right\rangle=-2 .
$$

From (4.1.8-4.1.9) we get that

$$
\phi^{*} h_{g}=h_{g} .
$$

Now we determine the action of $\phi^{*}$ on the remaining part of $H^{2}\left(S^{[g-1]}\right)$. It suffices to prove that (4.1.7) holds for $\left(S, D_{2 g-2}\right) \in U$ such that (4.1.10) holds. Since (1.0.2) is a homomorphiam $H^{2}(\phi)$ is an isometric involution; by (4.1.11) and (4.1.13) we get that the restriction of $H^{2}(\phi)$ to $H_{\mathbb{Q}}^{1,1}\left(S^{[g-1]}\right)$ is either the identity or the reflection $\mathbb{Q} h_{g}$. To show that the latter holds it suffices to check that

$$
\phi^{*} c_{1}\left(\Delta_{g-1}\right) \neq c_{1}\left(\Delta_{g-1}\right) .
$$

By (4.1.12) any effective divisor homologous to $\Delta_{g-1}$ must be equal to $\Delta_{g-1}$; since $\phi^{*}\left(\Delta_{g-1}\right) \neq \Delta_{g-1}$ we get 4.1.14). Now consider $T\left(S^{[g-1]}\right):=H_{\mathbb{Q}}^{1,1}\left(S^{[g-1]}\right)^{\perp}$ : it is left invariant by the isometry $H^{2}(\phi)$. To finish the proof it will suffice to show that the restriction of $H^{2}(\phi)$ to $T\left(S^{[g-1]}\right)$ is equal to $(-1)$. Since the eigenspaces of the restriction of $H^{2}(\phi)$ to $T\left(S^{[g-1]}\right)$ are Hodge substructures and $T\left(S^{[g-1]}\right)$ has no non-trivial Hodge substructures, the restriction of $H^{2}(\phi)$ to $T\left(S^{[g-1]}\right)$ is equal to \pm 1 . Thus it suffices to show that

$$
\phi^{*}\left(\sigma^{[g-1]}\right)=-\sigma^{[g-1]}
$$

where $\sigma^{[g-1]}$ is the symplectic form on $S^{[g-1]}$ induced by a symplectic form $\sigma$ on $S$ (see [3], p. 766). Letting $\phi([Z])=[W]$ we have

$$
Z+W \sim D_{2 g-2} \cdot D_{2 g-2},
$$

and hence Equality (4.1.15) follows from Mumford's Theorem on 0-cycles (see Prop (22.24) of 34). 


\subsubsection{More on the involution}

Let $f_{S}: S \rightarrow\left|D_{2 g-2}\right|^{\vee}$ be the natural map and let $H_{g}$ be the divisor class on $S^{[g-1]}$ such that $c_{1}\left(H_{g}\right)=h_{g}$. Suppose first that $g=3$, and consider the three possible cases

(a) $D_{4}$ is very ample and $\operatorname{Im}\left(f_{S}\right)$ does not contain lines,

(b) $D_{4}$ is very ample and $\operatorname{Im}\left(f_{S}\right)$ does contain lines $\ell_{1}, \ldots, \ell_{k}$,

(c) $D_{4}$ is not very ample, i.e. $f_{S}$ is 2 -to- 1 onto a quadric.

In case (a) the divisor class $H_{3}$ is ample. Furthermore $\phi$ is regular (Proposition (11) of [4]). Thus $H^{2}(\phi)$ is constant on the open subset parametrizing $\left(S, D_{2 g-2}\right)$ for which (a) holds; by Proposition (4.1) we get that $H^{2}(\phi)$ is the reflection in $\mathbb{Z} h_{3}$. Applying Corollary (3.2) we get that $\phi$ extends to all small deformations of $S^{[2]}$ keeping $h_{3}$ of type $(1,1)$ - notice that the generic such deformation is not of the type $(K 3)^{[2]}$. In case (b) the divisor class $H_{3}$ is globally generated and big but not ample. Furthermore $\phi$ is not regular (Proposition (11) of 4]). Beauville shows that to resolve the indeterminacies of $\phi$ it suffices to blow up $\ell_{1}^{(2)} \cup \cdots \cup \ell_{k}^{(2)}$, and in fact $\phi$ lifts to a regular involution on the blow-up: thus $\phi$ is the flop of $\ell_{1}^{(2)} \cup \cdots \cup \ell_{k}^{(2)}$. As is easily checked $H^{2}(\phi)$ is the reflection in $\mathbb{Z} h_{3}$. In case (c) the map $\phi$ is not regular, in particular $H_{3}$ is not ample. Furthermore $H^{2}(\phi)$ is not the reflection in $\mathbb{Z} h_{3}$. Now consider $g \geq 4$ : then the map $\phi$ is never regular (p. 24 of [4), in particular $H_{g}$ is not ample. If $g=4,5$ and $S$ is generic then Beauville (p. 25 of [4]) shows that the indeterminacies of $\phi$ are resolved by a single blow-up with smooth center ( $\phi$ is a Mukai elementary modification [27]).

\subsection{Mukai reflections}

These are involutions of moduli spaces of sheaves on a $K 3$ surface. Beauville's involutions are never regular in dimension greater than 4: on the other hand Mukai reflections give examples of regular involutions on deformations of $(K 3)^{[n]}$ for arbitrary $n$. The action of a Mukai reflection on $H^{2}$ is the reflection in a class of square 2 .

\subsubsection{Moduli of sheaves on a $K 3$ surface $S$}

We recall basic definitions and results. Let $F$ be a sheaf on $S$; following Mukai [26] one sets

$$
v(F):=\operatorname{ch}(F) \sqrt{\operatorname{Td}(S)}=\operatorname{ch}(F)(1+\eta) \in H^{*}(S ; \mathbb{Z}),
$$

where $\eta \in H^{4}(S ; \mathbb{Z})$ is the orientation class. For $\alpha \in H^{*}(S)$ with degree- $q$ component given by $\alpha_{q}$ we set

$$
\alpha^{\vee}:=\alpha_{0}-\alpha_{2}+\alpha_{4}
$$

On $H^{*}(S)$ we have Mukai's bilinear symmetric form defined by

$$
\langle u, w\rangle:=-\int_{S} u \wedge w^{\vee} .
$$


By Hirzebruch-Riemann-Roch we have

$$
\langle v(E), v(F)\rangle=-\chi(E, F):=-\sum_{i=0}^{2}(-1)^{i} \operatorname{dim} \operatorname{Ext}^{i}(E, F) .
$$

Let

$$
\mathbf{v}=r+\ell+s \eta \in H^{0}(S ; \mathbb{Z})_{\geq 1} \oplus H_{\mathbb{Z}}^{1,1}(S) \oplus H^{4}(S ; \mathbb{Z}) .
$$

Given an ample divisor $D$ on $S$ we let $\mathcal{M}(\mathbf{v})$ be the moduli space of GiesekerMaruyama $D$-semistable torsion-free sheaves $F$ on $S$ with $v(F)=\mathbf{v}$; this is a projective variety [12, 24]. An example: let $D_{2 g-2}$ be a divisor on $S$ with $D_{2 g-2} \cdot D_{2 g-2}=2 g-2$ and let

$$
\mathbf{v}:=1+c_{1}\left(D_{2 g-2}\right)+\eta .
$$

Then $\mathcal{M}(\mathbf{v})$ parametrizes sheaves $I_{Z}\left(D_{2 g-2}\right)$ where $Z$ is a length- $(g-1)$ subscheme of $S$, hence $\mathcal{M}(\mathbf{v})=S^{[g-1]}$. For the sake of simplicity we omit reference to $D$ in the notation for $\mathcal{M}(\mathbf{v})$; however one should keep in mind that if we change $D$ the moduli space $\mathcal{M}(\mathbf{v})$ might change. Assume that $F$ is stable and that $v(F)=\mathbf{v}$ : the tangent space of $\mathcal{M}(\mathbf{v})$ at the point $[F]$ corresponding to $F$ is canonically identified with $\operatorname{Ext}^{1}(F, F)$ (see Cor (4.5.2) of [16]). Stability of $F$ and Serre duality give

$$
1=\operatorname{dim} \operatorname{Hom}(F, F)=\operatorname{dim} \operatorname{Ext}^{2}(F, F),
$$

hence (4.2.3) gives

$$
\operatorname{dim} \operatorname{Ext}^{1}(F, F)=2+\langle\mathbf{v}, \mathbf{v}\rangle .
$$

By a theorem of Mukai [26] we know that $\mathcal{M}(\mathbf{v})$ is smooth at $[F]$ and thus

$$
\operatorname{dim}_{[F]} \mathcal{M}(\mathbf{v})=2+\langle\mathbf{v}, \mathbf{v}\rangle \quad \text { if } F \text { is stable. }
$$

It has been proved that under certain hypotheses on $D$ and $\mathbf{v}$ the moduli space $\mathcal{M}(\mathbf{v})$ is an irreducible symplectic variety deformation equivalent to $S^{[n]}$ where $2 n=2+\langle\mathbf{v}, \mathbf{v}\rangle$. In order to state a result which suffices for our purposes we give a definition.

Definition 4.3. Keep notation and assumptions as above. The ample divisor $D$ is $\mathbf{v}$-generic if there exists no couple $\left(r_{0}, \ell_{0}\right)$ consisting of an integer $0<r_{0}<r$ and $\ell_{0} \in H_{\mathbb{Z}}^{1,1}(S)$ such that

$$
\left(r \ell_{0}-r_{0} \ell\right) \cdot D=0, \quad-\left(r^{2}\langle\mathbf{v}, \mathbf{v}\rangle+2 r^{4}\right) \leq 4\left(r \ell_{0}-r_{0} \ell\right)^{2}<0 .
$$

Given $\mathbf{v}$ there exists a v-generic ample $D[30$. The following lemma is proved in 30 .

Lemma 4.4. Keep notation and assumptions as above. Assume that $r+\ell$ is indivisible and that $D$ is $\mathbf{v}$-generic. Let $F$ be a $D$-slope semistable sheaf with $v(F)=\mathbf{v}$; then $F$ is slope-stable. In particular $\mathcal{M}(\mathbf{v})$ is smooth.

The following theorem was proved by Yoshioka [35] (see [30] for the case when $\ell$ is indivisible). 
Theorem 4.5. [Yoshioka] Keep notation and assumptions as above. Assume that $r+\ell$ is indivisible and that $D$ is $\mathbf{v}$-generic. Then $\mathcal{M}(\mathbf{v})$ is an irreducible symplectic variety deformation equivalent to $S^{[n]}$ where $2 n=2+\langle\mathbf{v}, \mathbf{v}\rangle$.

Under these hypotheses there is a beautiful description of $H^{2}(\mathcal{M}(\mathbf{v}))$ and its Beauville form given by Mukai [29] and proved by Yoshioka 35] (see [30] for the case when $\ell$ is indivisible). First we need some preliminaries (see [26]). A quasi-family of sheaves on $S$ parametrized by $T$ with Mukai vector $\mathbf{v}$ consists of a sheaf $\mathcal{F}$ on $S \times T$ flat over $T$ with $\left.\mathcal{F}\right|_{S \times\{t\}} \cong F^{\oplus d}$ where $v(F)=\mathbf{v}$ and $d$ is some positive integer independent of $t$; we set $\sigma(\mathcal{F}):=d$. Two quasi-families $\mathcal{F}, \mathcal{G}$ on $S$ parametrized by $T$ with Mukai vector $\mathbf{v}$ are equivalent if there exist vector-bundles $\mathcal{V}, \mathcal{W}$ on $T$ such that $\mathcal{F} \otimes p_{T}^{*} \mathcal{V} \cong \mathcal{G} \otimes p_{T}^{*} \mathcal{W}$, where $p_{T}: S \times T \rightarrow T$ is the projection. Given a quasi-family $\mathcal{F}$ as above we let $\theta_{\mathcal{F}}: H^{*}(S) \rightarrow H^{2}(T)$ be defined by

$$
\theta_{\mathcal{F}}(\alpha):=\frac{1}{\sigma(\mathcal{F})} p_{T, *}\left[\operatorname{ch}(\mathcal{F})\left(1+p_{S}^{*} \eta\right) p_{S}^{*}\left(\alpha^{\vee}\right)\right]_{6}
$$

where $p_{S}, p_{T}: S \times T \rightarrow S, T$ are the projections and $[\cdots]_{q}$ denotes the component of $[\cdots]$ in $H^{q}(S \times T)$. An easy computation gives the following result.

Lemma 4.6. Keeping notation as above assume that $\mathcal{F}, \mathcal{G}$ are two equivalent quasi-families of sheaves on $S$ parametrized by $T$ with Mukai vector $\mathbf{v}$. If $\alpha \in \mathbf{v}^{\perp}$ then

$$
\theta_{\mathcal{F}}(\alpha)=\theta_{\mathcal{G}}(\alpha)
$$

Mukai (Thm. (A.5) of [26]) showed that there exists a tautological quasifamily $\mathcal{E}$ on $S$ parametrized by $\mathcal{M}(\mathbf{v})$ i.e. a quasi-family $\mathcal{E}$ of sheaves on $S$ parametrized by $\mathcal{M}(\mathbf{v})$ with Mukai vector $\mathbf{v}$ such that $\left.\mathcal{E}\right|_{S \times[F]} \cong F^{\oplus \sigma(\mathcal{F})}$. Furthermore the proof of Thm. (A.5) of [26] shows that any two tautological quasifamiles are equivalent. Thus by Lemma (4.6) we get a well-defined linear map

$$
\theta_{\mathbf{v}}: \mathbf{v}^{\perp} \longrightarrow H^{2}(\mathcal{M}(\mathbf{v}))
$$

by setting $\theta_{\mathbf{v}}:=\frac{1}{\sigma(\mathcal{F})} \theta_{\mathcal{F}}$ where $\mathcal{F}$ is any tautological quasi-family of sheaves on $S$ parametrized by $\mathcal{M}(\mathbf{v})$. Now define a weight-two Hodge structure on $H^{*}(S)$ by setting $F^{0}:=H^{*}(S), F^{1}:=H^{0}(S) \oplus F^{1} H^{2}(S) \oplus H^{4}(S)$ and $F^{2}:=F^{2} H^{2}(S)$. Since $\mathbf{v}$ is integral of type $(1,1)$ the orthogonal $\mathbf{v}^{\perp}$ inherits a lattice structure and a Hodge structure from $H^{*}(S)$. The following result was proved by Yoshioka 35. (see [30] for the case when $\ell$ is primitive and [26] for $\mathbf{v}$ isotropic).

Theorem 4.7. [Yoshioka] Keep notation and assumptionms as above. Suppose that $r+\ell$ is indivisible, that $\langle\mathbf{v}, \mathbf{v}\rangle \geq 2$ and that $D$ is $\mathbf{v}$-generic. Then $\theta_{\mathbf{v}}$ is an isomorphism of integral Hodge structures and defines an isometry between $\mathbf{v}^{\perp}$ and $\left(H^{2}(\mathcal{M}(\mathbf{v}) ; \mathbb{Z}),(\cdot, \cdot)\right)$.

An example: if $\mathbf{v}$ is given by (4.2.5) we get 4.1.3 with $n=g-1$.

\subsubsection{Definition of Mukai reflections}

Set $r=s$ in (4.2.4) i.e.

$$
\mathbf{v}=r+\ell+r \eta, \quad r \geq 1
$$


Under certain hypotheses there exists a Mukai reflection on $\mathcal{M}(\mathbf{v})$, i.e. a birational involution

$$
\phi_{\mathbf{v}}: \mathcal{M}(\mathbf{v}) \cdots>\mathcal{M}(\mathbf{v}) .
$$

We will make the following assumption.

Hypothesis 4.8. If $A$ is a divisor on $S$ the intersection number $A \cdot D$ is a multiple of $\ell \cdot D$.

Remark 4.9. If Hypothesis (4.8) holds then $\ell$ is indivisible and $D$ is $\mathbf{v}$-generic. By Theorem 4.5) we get that $\mathcal{M}(\mathbf{v})$ is a deformation of $S^{[n]}$ where $2 n=$ $\langle\mathbf{v}, \mathbf{v}\rangle+$,2 . If furthermore $\langle\mathbf{v}, \mathbf{v}\rangle \geq 2$ then by Theorem 4.8) the Hodge and lattice structures on $H^{2}(\mathcal{M}(\mathbf{v}))$ are isomorphic to those of $\mathbf{v}^{\perp}$.

We also add the following assumption:

$$
\ell \cdot D>0 .
$$

Let $[F] \in \mathcal{M}(\mathbf{v})$ : then

$$
h^{2}(F)=0, \quad \chi(F)=\chi\left(\mathcal{O}_{S}, F\right)=2 r .
$$

To get the first equality notice that $H^{2}(F) \cong \operatorname{Hom}\left(F, \mathcal{O}_{S}\right)^{\vee}$ by Serre duality and that by slope-semitability of $F$ we have $\operatorname{Hom}\left(F, \mathcal{O}_{S}\right)=0$. The second equality follows from 4.2.3). From (4.2.13) we get that

$$
h^{0}(F) \geq 2 r \text { for }[F] \in \mathcal{M}(\mathbf{v}) .
$$

Let

$$
\widetilde{F}:=\operatorname{Im}\left(H^{0}(F) \otimes \mathcal{O}_{S} \rightarrow F\right)
$$

be the subsehaf of $F$ generated by global sections.

Lemma 4.10. [Markman] Keep notation and hypotheses as above. Then

(1) $F / \widetilde{F}$ is Artinian.

(2) the sheaf E fitting into the exact sequence

$$
0 \rightarrow E \rightarrow H^{0}(F) \otimes \mathcal{O}_{S} \rightarrow \widetilde{F} \rightarrow 0
$$

is locally-free and slope-stable.

Proof. (1) follows from Lemma (3.5), p. 661 in [22. (2): $E$ is locally-free because $\widetilde{F}$ is a torsion-free sheaf on the smooth surface $S$ and hence its projective dimension is at most 1 . That $E$ is slope-stable is proved in 22], pp. 682-684 "The case $a+b+t>a$ ".

Now let $U(\mathbf{v}) ß \mathcal{M}(\mathbf{v})$ be the subset defined by

$$
U(\mathbf{v}):=\left\{[F] \in \mathcal{M}(\mathbf{v}) \mid h^{0}(F)=2 r\right\} .
$$

By (4.2.14) $U(\mathbf{v})$ is open in $\mathcal{M}(\mathbf{v})$. 
Lemma 4.11. [Markman] Keep notation and hypotheses as above. Then $U(\mathbf{v})$ is Zariski-dense in $\mathcal{M}(\mathbf{v})$. Furthermore $U(\mathbf{v})=\mathcal{M}(\mathbf{v})$ if

$$
\langle\mathbf{v}, \mathbf{v}\rangle \leq(4 r-2)
$$

otherwise

$$
\operatorname{cod}(\mathcal{M}(\mathbf{v}) \backslash U(\mathbf{v}), \mathcal{M}(\mathbf{v}))=2 r+1 .
$$

Proof. Follows from Corollary (3.16), p. 672 of 22. (Notice: the definition of $\mu(v)$ is on p. 628 , loc.cit.)

Let $U^{b}(\mathbf{v}) B U(\mathbf{v})$ be the open subset defined by

$$
U^{b}(\mathbf{v}):=\{[F] \in U(\mathbf{v}) \mid F \text { locally-free and globally generated }\} .
$$

Let $[F] \in U^{b}(\mathbf{v})$; the sheaf $E$ appearing in Exact Sequence 4.2.16) is locallyfree, slope-stable and $v(E)=v(F)^{\vee}$. Thus $\left[E^{\vee}\right] \in \mathcal{M}(\mathbf{v})$ and we have a regular map

$$
\begin{array}{ccc}
U^{b}(\mathbf{v}) & \longrightarrow \mathcal{M}(\mathbf{v}) \\
{[F]} & \mapsto & E^{\vee}
\end{array}
$$

Theorem 4.12. [Markman] Keep notation and hypotheses as above. There exists an anti-symplectic (see (3.0.1)) birational involution

$$
\phi_{\mathbf{v}}: \mathcal{M}(\mathbf{v}) \cdots>\mathcal{M}(\mathbf{v})
$$

with the following properties:

(1) $\phi_{\mathbf{v}}$ is regular on $U(\mathbf{v})$. In particular if 4.2 .18$)$ holds then $\phi_{\mathbf{v}}$ is a regular involution.

(2) The restriction of $\phi_{\mathbf{v}}$ to $U^{\mathrm{b}}(\mathbf{v})$ coincides with the map given by 4.2.21).

(3) $\phi_{\mathbf{v}}\left(U^{b}(\mathbf{v})\right)=U^{b}(\mathbf{v})$.

Proof. In the notation of 22 the map $\phi_{\mathbf{v}}$ is $\widetilde{q}_{0}$ of Theorem (3.21), p. 681, with $a=b=r$ and $\mathcal{L}$ the line-bundle such that $c_{1}(\mathcal{L})=\ell$. Markman does not prove that $\phi_{\mathbf{v}}$ is anti-symplectic. If $r \geq 2$ this follows from Proposition (4.14) below. If $r=1$ the map $\phi_{\mathbf{v}}$ is Beauville's involution and hence it is anti-symplectic by Proposition (4.1). (1) is Item (1) of Theorem (3.21), in 22] (the case $t=0$ ). (2) is in 22, first line of p. 683 . To prove (3) it suffices to show that

$$
\phi_{\mathbf{v}}\left(U^{\mathrm{b}}(\mathbf{v})\right) B U^{\mathrm{b}}(\mathbf{v})
$$

because $\phi_{\mathbf{v}}^{-1}=\phi_{\mathbf{v}}$. Let $[F] \in U^{\mathrm{b}}(\mathbf{v})$ and let $E$ be the sheaf appearing in (4.2.16): we must show that $\left[E^{\vee}\right] \in U^{\mathrm{b}}(\mathbf{v})$. We know by Item (2) of Lemma (4.10) that $E^{\vee}$ is locally-free. Applying the $\operatorname{Hom}\left(, \mathcal{O}_{S}\right)$-functor to (4.2.16) we get a sequence

$$
0 \rightarrow F^{\vee} \rightarrow H^{0}(F)^{\vee} \otimes \mathcal{O}_{S} \rightarrow E^{\vee} \rightarrow 0
$$

which is exact because $F=\widetilde{F}$ is locally-free. Thus $E^{\vee}$ is globally-generated. Since $[F] \in U(\mathbf{v})$ we have $H^{1}\left(F^{\vee}\right)=H^{1}(F)^{\vee}=0$ and hence the long exact cohomology sequence associated to (4.2.23) gives $h^{0}\left(E^{\vee}\right)=2 r$. This proves (4.2.22). 
Lemma 4.13. Keep notation and hypotheses as above and assume furthermore that $r \geq 2$. Then $U^{b}(\mathbf{v})$ is Zariski-dense in $\mathcal{M}(\mathbf{v})$.

Proof. Let $\Delta(\mathbf{v}) B \mathcal{M}(\mathbf{v})$ be given by

$$
\Delta(\mathbf{v}):=\left\{[F] \in \mathcal{M}(\mathbf{v}) \mid F^{\vee \vee} \neq F\right\}
$$

i.e. the (closed) subset parametrizing singular (not locally-free) sheaves. Let

$$
\Theta^{0}(\mathbf{v}):=\{[F] \in U(\mathbf{v}) \mid \widetilde{F} \neq F\}
$$

i.e. the (closed) subset in $U(\mathbf{v})$ parametrizing sheaves which are not globally generated. By Exact Sequence (104), p. 683 in 22 .

$$
\phi_{\mathbf{v}}\left(\Theta^{0}(\mathbf{v})\right)=\Delta(\mathbf{v}) \cap U(\mathbf{v}) .
$$

Since $r \geq 2$ we know that $\Delta(\mathbf{v})$ is a proper subset of $\mathcal{M}(\mathbf{v})$ (see also (4.17)). Thus $\Theta^{0}(\mathbf{v})$ is a proper subset of $\mathcal{M}(\mathbf{v})$. Since $U^{b}(\mathbf{v})=U(\mathbf{v}) \backslash \Theta^{0}(\mathbf{v}) \backslash \Delta(\mathbf{v})$ we are done.

Lemma (4.11) and Theorem (4.12) allow us to produce many moduli spaces $\mathcal{M}(\mathbf{v})$ with a regular anti-symplectic involution. An example: let $(S, D)$ be a degree- $(2 g-2)$ polarized $K 3$ and set

$$
\mathbf{v}:=r+c_{1}(D)+r \eta, \quad g \leq r^{2}+2 r .
$$

Choosing $D$ as the ample divisor defining (semi)stability of sheaves we see that the hypotheses of Lemma (4.11) and Theorem (4.12) are satisfied except possibly Hypothesis (4.8). For $(S, D)$ contained in an open dense subset of the moduli space of degree- $(2 g-2)$ polarized $K 3$ 's Hypothesis (4.8) is satisfied as well and hence $\phi_{\mathbf{v}}$ is a regular involution of $\mathcal{M}(\mathbf{v})$. Notice that we get examples in any (even) dimension.

\subsubsection{Description of $H^{2}\left(\phi_{\mathbf{v}}\right)$ and applications}

Throughout this subsubsection we assume that $\mathbf{v}$ is given by (4.2.10) with $r \geq 2$ and that both Hypothesis (4.8) and (4.2.12) hold. By Remark (4.9) we know that $\mathcal{M}(\mathbf{v})$ is a deformation of $(K 3)^{[n]}$ where $2 n=2+\langle\mathbf{v}, \mathbf{v}\rangle$, and furthermore Theorem (4.12) gives us the birational involution $\phi_{\mathbf{v}}: \mathcal{M}(\mathbf{v}) \cdots>\mathcal{M}(\mathbf{v})$. We also assume that $\langle\mathbf{v}, \mathbf{v}\rangle \geq 2$ i.e. that $\operatorname{dim} \mathcal{M}(\mathbf{v}) \geq 4$. By Remark (4.9) we know that $\theta_{\mathbf{v}}: \mathbf{v}^{\perp} \rightarrow H^{2}(\mathcal{M}(\mathbf{v}))$ is an isomorphism of lattices (and Hodge structures). Since $(\eta-1) \in \mathbf{v}^{\perp}$ it makes sense to set $h_{\mathbf{v}}:=\theta_{\mathbf{v}}(\eta-1)$; notice that $\left(h_{\mathbf{v}}, h_{\mathbf{v}}\right)=2$. The following result extends to higher rank the formula of Proposition (4.1) (notice that if $\mathbf{v}$ is given by (4.2.5) then $\theta_{\mathbf{v}}(\eta-1)=h_{g}$ where $h_{g}$ is as in (4.1.6)).

Proposition 4.14. Keep notation and hypotheses as above. Then $H^{2}\left(\phi_{\mathbf{v}}\right)$ is the reflection in the span of $h_{\mathbf{v}}$, i.e.

$$
H^{2}\left(\phi_{\mathbf{v}}\right)(\alpha)=-\alpha+\left(\alpha, h_{\mathbf{v}}\right) h_{\mathbf{v}} .
$$

Before proving the proposition we give a corollary. Let $H_{\mathbf{v}}$ be the divisor class such that $c_{1}\left(H_{\mathbf{v}}\right)=h_{\mathbf{v}}$. 
Corollary 4.15. Keep notation and hypotheses as above, and suppose furthermore that $\langle\mathbf{v}, \mathbf{v}\rangle \leq(4 r-2)$, i.e. that $\operatorname{dim} \mathcal{M}(\mathbf{v}) \leq 4 r$. Then:

(1) $\phi_{\mathbf{v}}$ extends to all small deformations of $\mathcal{M}(\mathbf{v})$ that keep $h_{\mathbf{v}}$ of type $(1,1)$,

(2) $H_{\mathbf{v}}$ is ample.

Proof. (1): The map $\phi_{\mathbf{v}}$ is regular by Item (1) of Theorem (4.12) and thus Item (1) follows from Proposition (4.14) and Corollary (3.2). (2): Let $H_{0}$ be an ample divisor on $\mathcal{M}(\mathbf{v})$ - it exists because $\mathcal{M}(\mathbf{v})$ is projective. Since $\phi_{\mathbf{v}}$ is regular $\phi_{\mathbf{v}}^{*} H_{0}$ is ample. Thus $\left(H_{0}+\phi_{\mathbf{v}}^{*} H_{0}\right)$ is an ample divisor class invariant for $\phi_{\mathbf{v}}^{*}$. By Proposition (4.14) $\left(H_{0}+\phi_{\mathbf{v}}^{*} H_{0}\right)$ is a multiple of $H_{\mathbf{v}}$; thus either $H_{\mathbf{v}}$ or $\left(-H_{\mathbf{v}}\right)$ is ample. Suppose that $\left(-H_{\mathbf{v}}\right)$ is ample: we will arrive at a contradiction. Let $\mu_{\mathbf{v}}: H^{2}(S) \rightarrow H^{2}(\mathcal{M}(\mathbf{v}))$ be Donaldson's map (see [30]) and $L$ be the line-bundle on $S$ such that $c_{1}(L)=\ell$. By Hypothesis (4.8) and 4.2.12) $L$ is big and nef and hence

$$
\int_{\mathcal{M}(\mathbf{v})} c_{1}\left(-H_{\mathbf{v}}\right)^{2 n-1} \wedge \mu_{\mathbf{v}}(\ell)>0
$$

where $2 n=\operatorname{dim} \mathcal{M}(\mathbf{v})$. By Fujiki's Formula (1.0.1) we get that

$$
\left(-H_{\mathbf{v}}, \mu_{\mathbf{v}}(\ell)\right)>0 .
$$

On the other hand the second-to-last formula on p. 639 of 30 (warning: the map $\theta_{\mathbf{v}}$ in [30] is the opposite of $\theta_{\mathbf{v}}$ of the present paper) gives that

$$
\left(-H_{\mathbf{v}}, \mu_{\mathbf{v}}(\ell)\right)=-1 / r \int_{S} \ell \cdot \ell<0 .
$$

This contradicts (4.2.27); thus $\left(-H_{\mathbf{v}}\right)$ is not ample and hence $H_{\mathbf{v}}$ must be ample.

Before proving Proposition (4.14) we prove some lemmas. The first lemma is very similar to Theorem (2.9) of [35].

Lemma 4.16. Let $U^{\mathrm{b}}(\mathbf{v})$ be as in 4.2.20) and $\iota_{\mathbf{v}}: U^{\mathrm{b}}(\mathbf{v}) \hookrightarrow \mathcal{M}(\mathbf{v})$ be the inclusion. Then

$$
\iota_{\mathbf{v}}^{*} \circ H^{2}\left(\phi_{\mathbf{v}}\right)=\iota_{\mathbf{v}}^{*} \circ R_{h_{\mathbf{v}}} .
$$

Proof. Let $\phi_{\mathbf{v}}^{\mathrm{b}}: U^{\mathrm{b}}(\mathbf{v}) \rightarrow U^{\mathrm{b}}(\mathbf{v})$ be the restriction of $\phi_{\mathbf{v}}$ (see Item (3) of Theorem (4.12) and $\Phi_{\mathbf{v}}^{b}:=\operatorname{Id}_{S} \times \phi_{\mathbf{v}}^{b}$. Let $\mathcal{F}$ be the restriction to $S \times U^{\mathrm{b}}(\mathbf{v})$ of a quasi-tautological family on $S \times \mathcal{M}(\mathbf{v})$. Let $R_{(\eta-1)}: H^{*}(S) \rightarrow H^{*}(S)$ be the reflection in the span of $(\eta-1)$ i.e.

$$
R_{(\eta-1)}(\alpha):=-\alpha+\langle\alpha, \eta-1\rangle(\eta-1) .
$$

$\mathbf{v}^{\perp}$ is mapped to itself by $R_{(\eta-1)}$ because $R_{(\eta-1)}(\mathbf{v})=-\mathbf{v}$. Given $\theta_{\mathbf{v}}(\alpha) \in$ $H^{2}(\mathcal{M}(\mathbf{v}))$, where $\alpha \in \mathbf{v}^{\perp}$, we have

$$
\begin{aligned}
\iota_{\mathbf{v}}^{*} \circ H^{2}\left(\phi_{\mathbf{v}}\right)\left(\theta_{\mathbf{v}}(\alpha)\right) & =\frac{1}{\sigma(\mathcal{F})} \theta_{\left(\Phi_{\mathbf{v}}^{\mathrm{b}}\right) * \mathcal{F}}(\alpha) \\
\iota_{\mathbf{v}}^{*} \circ R_{h_{\mathbf{v}}}\left(\theta_{\mathbf{v}}(\alpha)\right) & =\frac{1}{\sigma(\mathcal{F})} \theta_{\mathcal{F}}\left(R_{(\eta-1)}(\alpha)\right),
\end{aligned}
$$


hence we must prove that

$$
\theta_{\left(\Phi_{\mathbf{v}}^{\mathrm{b}}\right) * \mathcal{F}}(\alpha)=\theta_{\mathcal{F}}\left(R_{(\eta-1)}(\alpha)\right)
$$

Let $\rho: S \times U^{\mathrm{b}}(\mathbf{v}) \rightarrow U^{\mathrm{b}}(\mathbf{v})$ be the projection. By definition of $U^{\mathrm{b}}(\mathbf{v})$ and Equation (4.2.13) we have

$$
R^{q} \rho_{*} \mathcal{F}=0, \quad q>0,
$$

hence $\rho_{*} \mathcal{F}$ is locally-free of rank $2 r \sigma(\mathcal{F})$. By definition of $U^{\mathrm{b}}(\mathbf{v})$ the natural map $\rho^{*}\left(\rho_{*} \mathcal{F}\right) \rightarrow \mathcal{F}$ is surjective. Let $\mathcal{E}$ be the sheaf on $S \times U^{b}(\mathbf{v})$ fitting into the exact sequence

$$
0 \rightarrow \mathcal{E} \rightarrow \rho^{*}\left(\rho_{*} \mathcal{F}\right) \rightarrow \mathcal{F} \rightarrow 0
$$

Let $[F] \in U^{b}(\mathbf{v})$ and $[G]=\phi_{\mathbf{v}}([F])$. By definition of $\phi_{\mathbf{v}}$ we have $\left.\mathcal{E}^{\vee}\right|_{S \times[F]} \cong$ $G^{\sigma(\mathcal{F})}$ and hence the quasi-families $\mathcal{E}^{\vee},\left(\Phi_{\mathbf{v}}^{b}\right)^{*} \mathcal{F}$ of sheaves on $S \times U^{b}(\mathbf{v})$ with Mukai vector $\mathbf{v}$ are equivalent. By Lemma (4.6) we get that

$$
\theta_{\left(\Phi_{\mathbf{v}}^{\mathrm{b}}\right) * \mathcal{F}}(\alpha)=\theta_{\mathcal{E} \vee}(\alpha), \quad \alpha \in \mathbf{v}^{\perp} .
$$

By definition of $U^{b}(\mathbf{v})$ the sheaf $\mathcal{F}$ is locally-free; taking the dual of (4.2.31) we get that

$$
\theta_{\mathcal{E} \vee}(\alpha)=\rho_{*}\left[\rho^{*} \operatorname{ch}\left(\rho_{*} \mathcal{F}\right)^{\vee} \pi^{*}\left((1+\eta) \alpha^{\vee}\right)\right]_{6}-\rho_{*}\left[\operatorname{ch}(\mathcal{F})^{\vee} \pi^{*}\left((1+\eta) \alpha^{\vee}\right)\right]_{6}
$$

where $\pi: S \times U^{b}(\mathbf{v}) \rightarrow S$ is the projection. By (4.2.30) we have $\rho_{!}(\mathcal{F})=\rho_{*}(\mathcal{F})$ hence Grothendieck-Riemann-Roch gives that

$$
c_{1}\left(\rho_{*} \mathcal{F}\right)=c_{1}\left(\rho_{!}(\mathcal{F})\right)=\rho_{*}\left[\operatorname{ch}(\mathcal{F}) \pi^{*}(1+2 \eta)\right]_{6}=\theta_{\mathcal{F}}(1+\eta) .
$$

Thus

$$
\begin{aligned}
\rho_{*}\left[\rho^{*} \operatorname{ch}\left(\rho_{*} \mathcal{F}\right)^{\vee} \pi^{*}\left((1+\eta) \alpha^{\vee}\right)\right]_{6} & =-\rho_{*}\left[\pi^{*}\left((1+\eta) \alpha^{\vee}\right)\right]_{4} c_{1}\left(\rho_{*} \mathcal{F}\right) \\
& =\theta_{\mathcal{F}}(\langle\alpha, 1+\eta\rangle(1+\eta)) .
\end{aligned}
$$

On the other hand

$$
\rho_{*}\left[\operatorname{ch}(\mathcal{F})^{\vee} \pi^{*}\left((1+\eta) \alpha^{\vee}\right)\right]_{6}=-\theta_{\mathcal{F}}\left(\alpha^{\vee}\right) .
$$

Plugging (4.2.35)-(4.2.36) into (4.2.33) we get that

$$
\theta_{\mathcal{E} \vee}(\alpha)=\theta_{\mathcal{F}}\left(\alpha^{\vee}+\langle\alpha, 1+\eta\rangle(1+\eta)\right) .
$$

By 4.2.32

$$
\theta_{\left(\Phi_{\mathbf{v}}^{b}\right) * \mathcal{F}}(\alpha)=\theta_{\mathcal{F}}\left(\alpha^{\vee}+\langle\alpha, 1+\eta\rangle(1+\eta)\right) .
$$

Since $R_{(\eta-1)}(\alpha)=\alpha^{\vee}+\langle\alpha, 1+\eta\rangle(1+\eta)$ this proves 4.2.29).

Lemma 4.17. Let $\Delta(\mathbf{v})$ be given by 4.2.24) and $\Theta(\mathbf{v})$ be the closure of 4.2.25).

(1) Both $\Delta(\mathbf{v})$ and $\Theta(\mathbf{v})$ are irreducible of codimension $(r-1)$.

(2) $\Delta(\mathbf{v}) \neq \Theta(\mathbf{v})$. 
Proof. (1): It is well-known that $\Delta(\mathbf{v})$ is irreducible of codimension $(r-1)$ : it follows from the fact that for any $\mathbf{w} \in H^{*}(S)$ the moduli space $\mathcal{M}(\mathbf{w})$ is either empty or of the expected dimension and by irreducibility of the Quotscheme parametrizing length- $q$ quotients of a fixed locally-free sheaf on $S$ (Theorem (6.A.1) of [16]). One also gets that the generic $F$ parametrized by $\Delta(\mathbf{v})$ fits into an exact sequence

$$
0 \rightarrow F \rightarrow E \stackrel{g}{\rightarrow} \mathbb{C}_{p} \rightarrow 0,
$$

where $[E] \in \mathcal{M}(\mathbf{v}+\eta)$ is generic and locally-free, $\mathbb{C}_{p}$ is the skyscraper sheaf at an arbitrary $p \in S$ and $g$ is an arbitrary surjection. From (4.2.26) we get that also $\Theta(\mathbf{v})$ is irreducible of codimension $(r-1)$. (2): We must prove that

$$
\text { if }[F] \in \Delta(\mathbf{v}) \text { is generic then } F \text { is globally generated. }
$$

Such an $F$ fits into (4.2.37) where $[E] \in \mathcal{M}(\mathbf{v}+\eta)$ is generic and locallyfree. We claim that $E$ is globally generated. The proof is analogous to that of Lemma (4.13). For $[E]$ generic $h^{0}(E)=2 r+1$ by Corollary (3.16), p. 672 of 22 : let $\widetilde{E} \beta E$ be the subsehaf of $E$ generated by global sections, then $E / \widetilde{E}$ is Artinian by Lemma (3.5) of [22]. One considers the Mukai map

$$
\begin{array}{ccc}
\mathcal{M}(\mathbf{v}+\eta) & \cdots> & M(1+\mathbf{v}) \\
{[E]} & \mapsto & {\left[G^{\vee}\right]}
\end{array}
$$

where $G$ is the sheaf fitting into the exact sequence

$$
0 \rightarrow G \rightarrow H^{0}(E) \otimes \mathcal{O}_{S} \rightarrow \widetilde{E} \rightarrow 0 .
$$

A parameter count shows that $\widetilde{E}=E$ for a generic $[E] \in \mathcal{M}(\mathbf{v}+\eta)$, we leave the details to the reader. Let $[E] \in \mathcal{M}(\mathbf{v}+\eta)$ be generic, let $\pi: \mathbb{P}\left(E^{\vee}\right) \rightarrow S$ be the projection and $\xi$ be the tautological line sub-bundle of $\pi^{*} E^{\vee}$. Since $H^{0}(E)=H^{0}\left(\xi^{\vee}\right)$ we know that the linear system $\left|\xi^{\vee}\right|$ has no base-locus and hence we have a regular map

$$
f: \mathbb{P}\left(E^{\vee}\right) \rightarrow \mathbb{P}\left(H^{0}(E)^{\vee}\right) \cong \mathbb{P}^{2 r} .
$$

Let $g \in E_{p}^{\vee}$ be the map appearing in (4.2.37) and let $x=[g]$; thus $x \in \mathbb{P}\left(E^{\vee}\right)$. If $d f(x)$ is injective the sheaf $F$ appearing in (4.2.37) is globally generated. Thus to prove 4.2.38) it suffices to show that $\operatorname{dim} \operatorname{Im}(f)=\operatorname{dim} \mathbb{P}\left(E^{\vee}\right)=(r+1)$ : this follows from the easily computed formula

$$
\int_{\mathbb{P}\left(E^{\vee}\right)} c_{1}\left(\xi^{\vee}\right)^{r+1}=\frac{1}{2}(\ell \cdot \ell)+1
$$

Let $U^{\sharp}(\mathbf{v}) ß U(\mathbf{v})$ be given by

$$
U^{\sharp}(\mathbf{v}):=\left\{[F] \in U(\mathbf{v}) \mid \ell\left(F^{\vee \vee} / F\right)+\ell(F / \widetilde{F}) \leq 1\right\} .
$$

This is an open subset of $\mathcal{M}(\mathbf{v})$ because both

$$
[F] \mapsto \ell\left(F^{\vee \vee} / F\right) \quad \text { and } \quad[F] \mapsto \ell(F / \widetilde{F})
$$

are upper semicontinuous functions on the open $U(\mathbf{v})$. 
Lemma 4.18. Keep notation and assumptions as above. Then

$$
\operatorname{cod}\left(\mathcal{M}(\mathbf{v}) \backslash U^{\sharp}(\mathbf{v}), \mathcal{M}(\mathbf{v})\right) \geq 2 .
$$

Proof. By Lemma (4.11) we know that $\operatorname{cod}(\mathcal{M}(\mathbf{v}) \backslash U(\mathbf{v}), \mathcal{M}(\mathbf{v})) \geq 2$ and hence it suffices to show that $\operatorname{cod}\left(U(\mathbf{v}) \backslash U^{\sharp}(\mathbf{v}), \mathcal{M}(\mathbf{v})\right) \geq 2$. We have a decomposition $\left(U(\mathbf{v}) \backslash U^{\sharp}(\mathbf{v})\right)=A_{(2,0)} \cup A_{(1,1)} \cup A_{(0,2)}$ where

$$
\begin{aligned}
& A_{(2,0)}:=\left\{[F] \in U(\mathbf{v}) \mid \ell\left(F^{\vee \vee} / F\right) \geq 2\right\}, \\
& A_{(1,1)}:=\left\{[F] \in U(\mathbf{v}) \mid \ell\left(F^{\vee \vee} / F\right) \geq 1, \ell(F / \widetilde{F}) \geq 1\right\}, \\
& A_{(0,2)}:=\{[F] \in U(\mathbf{v}) \mid \ell(F / \widetilde{F}) \geq 2\} .
\end{aligned}
$$

$A_{(2,0)}$ is a proper subset of $\Delta(\mathbf{v})$, see [4.2.37), and hence $A_{(2,0)}$ has codimension at least 2 by Lemma (4.17). $A_{(1,1)} \beta \Delta(\mathbf{v}) \cap \Theta(\mathbf{v})$ and hence it has codimension at least 2 by Lemma (4.17). Finally by (108) on p. 683 of [22 we have $\phi_{\mathbf{v}}\left(A_{(0,2)}\right) B A_{(2,0)}$ and hence $A_{(0,2)}$ has codimension at least 2 .

Proof of Proposition (4.14). First we prove the proposition in the case $r \geq 3$. Since $U^{b}(\mathbf{v})=U(\mathbf{v}) \backslash \Theta(\mathbf{v}) \backslash \Delta(\mathbf{v})$ we get from Item (1) of Lemma (4.17) and Lemma (4.11) that

$$
\operatorname{cod}\left(\mathcal{M}(\mathbf{v}) \backslash U^{b}(\mathbf{v}), \mathcal{M}(\mathbf{v})\right) \geq r-1 .
$$

Hence if $r \geq 3$ the map $H^{2}\left(\iota_{\mathbf{v}}\right)$ is an isomorphism, and thus Proposition (4.14) follows from Lemma (4.16). We are left with the case $r=2$, i.e.

$$
\mathbf{v}=2+\ell+2 \eta \text {. }
$$

Let $j_{\mathbf{v}}: U^{\sharp}(\mathbf{v}) \hookrightarrow \mathcal{M}(\mathbf{v})$ be the inclusion. The restriction of $\phi_{\mathbf{v}}$ to $U^{\sharp}(\mathbf{v})$ is an involution $\phi_{\mathbf{v}}^{\sharp}$ of $U^{\sharp}(\mathbf{v})$ : this follows from [22], p. 683. Let $\Phi_{\mathbf{v}}^{\sharp}:=\operatorname{Id}_{S} \times \phi_{\mathbf{v}}^{\sharp}$. Let $\mathcal{F}$ be the restriction to $S \times U^{\sharp}(\mathbf{v})$ of a quasi-tautological family on $S \times \mathcal{M}(\mathbf{v})$. Then

$$
j_{\mathbf{v}}^{*} \circ H^{2}\left(\phi_{\mathbf{v}}\right)\left(\theta_{\mathbf{v}}(\alpha)\right)=\theta_{\left(\Phi_{\mathbf{v}}^{\sharp}\right)^{*} \mathcal{F}}(\alpha), \quad \alpha \in \mathbf{v}^{\perp} .
$$

Let's construct a quasi-family equivalent to $\left(\Phi_{\mathbf{v}}^{\sharp}\right)^{*} \mathcal{F}$. Let $\widetilde{\mathcal{F}}$ be the sheaf on $S \times U^{\sharp}(\mathbf{v})$ given by

$$
\widetilde{\mathcal{F}}:=\operatorname{Im}\left(\rho^{*}\left(\rho_{*} \mathcal{F}\right) \rightarrow \mathcal{F}\right),
$$

where $\rho: S \times U^{\sharp}(\mathbf{v}) \rightarrow U^{\sharp}(\mathbf{v})$ is the projection. Let $\mathcal{E}$ be the sheaf on $S \times U^{\sharp}(\mathbf{v})$ fitting into the exact sequence

$$
0 \rightarrow \mathcal{E} \rightarrow \rho^{*}\left(\rho_{*} \mathcal{F}\right) \rightarrow \widetilde{\mathcal{F}} \rightarrow 0
$$

As is easily checked $\mathcal{E}$ is a quasi-family of torsion-free sheaves on $S$ parametrized by $U^{\sharp}(\mathbf{v})$ with Mukai vector $\mathbf{v}^{\vee}$. If $[F] \in U^{\sharp}(\mathbf{v})$ then $\left.\mathcal{E}\right|_{S \times[F]} \cong\left(E^{\prime}\right)^{\sigma(\mathcal{F})}$ where the double dual of $E^{\prime}$ is isomorphic to the sheaf $E$ of [4.2.16). $E^{\prime}$ is slope-stable by Item (2) of Lemma (4.10), and furthermore if $[F] \notin(\Theta(\mathbf{v}) \cup \Delta(\mathbf{v}))$ we have $\left[E^{\prime} \otimes L\right]=\phi_{\mathbf{v}}([F])$, where $L$ is the line-bundle on $S$ such that $c_{1}(L)=\ell$ : these facts imply that the quasi-families of sheaves on $S$ with Mukai vector $\mathbf{v}$ given by $\left(\Phi_{\mathbf{v}}^{\sharp}\right)^{*} \mathcal{F}$ and $\mathcal{E} \otimes \pi^{*} L$ are equivalent $\left(\pi: S \times U^{\sharp}(\mathbf{v}) \rightarrow S\right.$ is the projection). Let $\mathcal{G}:=\mathcal{E} \otimes \pi^{*} L$. By (4.2.39) and Lemma (4.6) we have

$$
j_{\mathbf{v}}^{*} \circ H^{2}\left(\phi_{\mathbf{v}}\right)\left(\theta_{\mathbf{v}}(\alpha)\right)=\theta_{\mathcal{G}}(\alpha), \quad \alpha \in \mathbf{v}^{\perp} .
$$


We compute the right-hand side. We have an exact sequence

$$
0 \rightarrow \widetilde{\mathcal{F}} \rightarrow \mathcal{F} \rightarrow \lambda \rightarrow 0
$$

where $l$ is a sheaf such that $\operatorname{supp}(l)$ is mapped by $\rho$ to $\Theta(\mathbf{v}) \cap U^{\sharp}(\mathbf{v})$ with finite fibers. By Lemma (4.17) $\Theta(\mathbf{v})$ is irreducible and hence

$$
\rho_{*} \operatorname{ch}_{3}(l)=j_{\mathbf{v}}^{*}\left(n c_{1}(\Theta(\mathbf{v}))\right), \quad n>0 .
$$

Let $\beta \in \mathbf{v}^{\perp} \cap H^{*}(S ; \mathbb{Z})$ be the class such that

$$
\theta_{\mathbf{v}}(\beta)=n c_{1}(\Theta(\mathbf{v})),
$$

and let $T_{\beta}: H^{*}(S) \rightarrow H^{*}(S)$ be defined by

$$
T_{\beta}(\alpha):=\alpha_{0} \beta-\left\langle e^{-\ell} \wedge \alpha, 1+\eta\right\rangle(1+\eta)-e^{-\ell} \wedge \alpha .
$$

Using (4.2.40), (4.2.42), (4.2.43) and (4.2.34) one gets that

$$
\theta_{\mathcal{G}}(\alpha)=\theta_{\mathcal{F}}\left(T_{\beta}(\alpha)\right) .
$$

Now notice that $T_{\beta}\left(\mathbf{v}^{\perp}\right)=\mathbf{v}^{\perp}$ (warning: $T_{\beta}$ is not an isometry!) and hence we can rewrite the above equation as

$$
\theta_{\mathcal{G}}(\alpha)=j_{\mathbf{v}}^{*} \circ \theta_{\mathbf{v}}\left(T_{\beta}(\alpha)\right) .
$$

By (4.18) the map $H^{2}\left(j_{\mathbf{v}}\right)$ is an isomorphism, hence (4.2.45) together with (4.2.41) gives that

$$
H^{2}\left(\phi_{\mathbf{v}}\right)\left(\theta_{\mathbf{v}}(\alpha)\right)=\theta_{\mathbf{v}}\left(T_{\beta}(\alpha)\right), \quad \alpha \in \mathbf{v}^{\perp} .
$$

Since (1.0.2) is a homomorphism $H^{2}\left(\phi_{\mathbf{v}}\right)$ is an isometric involution and hence the restriction of $T_{\beta}$ to $\mathbf{v}^{\perp}$ is an isometric involution. We claim that this implies that

$$
\beta=-(g-3)-\ell-2 \eta .
$$

Since with this value of $\beta$ we have $T_{\beta}=R_{(\eta-1)}$ the above equation proves Proposition (4.14) for $r=2$. Let's prove that (4.2.47) holds. Let $\mathrm{E}_{\mathbf{v}}:=(\mathbb{C} \oplus$ $\mathbb{C} \ell \oplus \mathbb{C} \eta) \cap \mathbf{v}^{\perp}$ and $\Xi_{\mathbf{v}}:=H^{2}(S) \cap \ell^{\perp} ;$ thus $\mathbf{v}^{\perp}=\mathrm{E}_{\mathbf{v}} \oplus_{\perp} \Xi_{\mathbf{v}}$. Since $T_{\beta}\left(\Xi_{\mathbf{v}}\right)=\Xi_{\mathbf{v}}$ we have $T_{\beta}\left(\mathrm{E}_{\mathbf{v}}\right)=\mathrm{E}_{\mathbf{v}}$; since $(1-\eta) \in \mathrm{E}_{\mathbf{v}}$ and $T_{\beta}(1-\eta) \in \beta+\mathrm{E}_{\mathbf{v}}$ we have $\beta \in \mathrm{E}_{\mathbf{v}}$. Let $\beta=b_{0}+b_{2} \ell+b_{4} \eta$ where $b_{i} \in \mathbb{Z}$ : thus

$$
T_{\beta}(\alpha)=\alpha_{0} b_{0}+\alpha_{0} b_{2} \ell+\alpha_{0} b_{4} \eta-\left\langle e^{-\ell} \wedge \alpha, 1+\eta\right\rangle(1+\eta)-e^{-\ell} \wedge \alpha .
$$

A straightforward computation shows that the restriction of the above map to $\mathbf{v}^{\perp}$ is an involution only if (4.2.47) holds.

It follows from (4.2.44) and (4.2.47) that

$$
c_{1}(\Theta(\mathbf{v}))=\theta_{\mathbf{v}}(-(g-3)-\ell-2 \eta) \quad \text { if } r=2 .
$$

By (4.2.26) we have $\Delta(\mathbf{v})=\phi_{\mathbf{v}}^{*} \Theta(\mathbf{v})$ and hence

$$
c_{1}(\Delta(\mathbf{v}))=\theta_{\mathbf{v}}(2+\ell+(g-3) \eta) \quad \text { if } r=2 .
$$




\subsection{Another example}

Let $S$ be a $K 3$ surface, $H_{S}$ be a divisor on $S$ with

$$
H_{S} \cdot H_{S}=2 g-2 .
$$

Let $h_{S}:=c_{1}\left(H_{S}\right)$. Let $\mu$ and $\xi_{2}$ be as in (4.1.1) and 4.1.3) respectively and let $h \in H_{\mathbb{Z}}^{1,1}\left(S^{[2]}\right)$ be

$$
h:=\mu\left(h_{S}\right)-t \xi_{2}
$$

If

$$
g=2+t^{2}
$$

then $(h, h)=2$ and hence the I Conjecture (1.3) predicts the existence of an antisymplectic birational involution of $S^{[2]}$. Let us test the conjecture for $t=0,1,2$. It suffices to produce the anti-symplectic involution under the hypothesis that $\left(S, H_{S}\right)$ is the generic couple with $S$ a $K 3$ and $H_{S}$ an ample degree- $(2 g-$ 2 ) divisor of a given divisibility. In fact once this is proved the degeneration procedure of Section (2) will give that the desired involution exists in general. Assume that $\left(S, H_{S}\right)$ is polarized and generic; thus by 23 the generic curve in $\left|H_{S}\right|$ is smooth and its genus is given by the $g$ appearing in (4.3.1). If $t=0$ then $g=2$ hence $S$ is a double cover of $\mathbb{P}^{2}$ and the covering involution $\phi_{S}: S \rightarrow S$ induces an anti-symplectic involution $\phi$ of $S^{[2]}$. Notice that $H^{2}(\phi)$ is never equal to $R_{h}$ (the reflection in $\mathbb{Z} h$ ) because $\phi^{*} \Delta_{2}=\Delta_{2}$ where $\Delta_{2}$ is as in (4.1.2). If $t=1$ then $g=3$. Let $\phi: S^{[2]} \rightarrow S^{[2]}$ be Beauville's involution defined in Subsection (4.1). By Proposition (4.1) we have $H^{2}(\phi)=R_{h}$ generically. (See also the comments at the end of Subsection (4.1).) Now let $t=2$. In this case we get a new example. Since $H_{S}$ is ample and $g=6$ we know by [23] that the linear system $\left|H_{S}\right|$ has no base-locus and that it defines an embedding $S \hookrightarrow\left|H_{S}\right|^{\vee} \cong \mathbb{P}^{6}$ as a degree-10 surface. According to Mukai [28] the generic $K 3$ surface of degree 10 in $\mathbb{P}^{6}$ is described as follows. Let $\mathbb{G}\left(1, \mathbb{P}^{4}\right) \beta \mathbb{P}^{9}$ be the Plücker embedding of the Grassmannian of lines in $\mathbb{P}^{4}$ and let

$$
F:=\mathbb{G}\left(1, \mathbb{P}^{4}\right) \cap \mathbb{P}^{6}
$$

where $\mathbb{P}^{6} \beta \mathbb{P}^{9}$ is a linear space transversal to $\mathbb{G}\left(1, \mathbb{P}^{4}\right)$. We have $K_{F} \cong \mathcal{O}_{F}(-2)$ and $\operatorname{deg} F=5$ i.e. $F$ is a Fano 3 -fold of index 2 and degree 5 ; by Iskovskih( $[17$, Thm. (4.2)) there is one isomorphism class of such Fano 3-folds. If $\bar{Q} \Omega \mathbb{P}^{6}$ is a quadric transversal to $F$ then

$$
S:=F \cap \bar{Q}
$$

is a $K 3$ surface of degree 10 in $\mathbb{P}^{6}$. Mukai (28, Cor. (4.3)) proved that the generic $K 3$ of degree 10 is given by (4.3.5). We will define an involution on $S^{[2]}$ analogous to Beauville's involution on $T^{[2]}$ where $T ß \mathbb{P}^{3}$ is a quartic surface: one replaces $\mathbb{P}^{3}$ by $F$ and $\mathbb{G}\left(1, \mathbb{P}^{3}\right)$ by the Hilbert scheme $W(F)$ parametrizing conics in $F$. Before defining the involution we state two results on lines and conics lying on $F$. Let $R(F)$ be the Hilbert scheme parametrizing lines contained in $F$. For $p \in F$ let

$$
R_{p}:=\{[L] \in R(F) \mid p \in L\} .
$$

The following result is due to Iskovskih. 
Lemma 4.19 (Cor. (6.6) of 17$)$ ). Keeping notation as above, $R(F)$ is isomorphic to $\mathbb{P}^{2}$.

For $Z \in\left(\mathbb{P}^{6}\right)^{[2]}$ we let $\langle Z\rangle \beta \mathbb{P}^{6}$ be the line spanned by $Z$. Let $B ß F^{[2]}$ be the closed subset given by

$$
B:=\left\{[Z] \in F^{[2]} \mid\langle Z\rangle ß F\right\} .
$$

For $[Z] \in F^{[2]}$ and $[\bar{L}] \in R(F)$ let

$$
\begin{aligned}
W_{Z} & :=\{[C] \in W(F) \mid Z ß C\}, \\
R_{\bar{L}} & :=\{[L] \in R(F) \mid[\bar{L}+L] \in W(F)\} .
\end{aligned}
$$

We will prove the following.

Lemma 4.20. Keep notation as above. Then

(1) Let $[\bar{L}] \in R(F)$. Then $R_{\bar{L}}$ is isomorphic to $\mathbb{P}^{1}$.

(2) Let $[Z] \in F^{[2]}$. If $[Z] \in B$ then $W_{Z}$ consists of those $[C] \in W(F)$ such that $C$ contains $\langle Z\rangle$ (hence $W_{Z}$ is identified with $R_{\langle Z\rangle}$ ). If $[Z] \notin B$ then $W_{Z}$ consists of a single conic.

(3) $W(F)$ is irreducible, rational of dimension 4.

Granting this lemma for the moment being, we proceed to define the involution. We make the following assumption regarding $S$ :

$$
S \text { contains no line and no conic. }
$$

Let $B_{S}:=B \cap S^{[2]}$ - this makes sense because we have an inclusion $S^{[2]} \beta F^{[2]}$. Let $U:=\left(S^{[2]} \backslash B_{S}\right) ; U$ is Zariski-dense in $S^{[2]}$ because $\operatorname{cod}\left(B_{S}, S^{[2]}\right)=2$ by Lemma (4.19). We will define a regular map

$$
\phi_{U}: U \rightarrow S^{[2]} .
$$

Let $[Z] \in U$. Since $[Z] \notin B_{S}$ there is a unique conic $C_{Z} \beta F$ containing $Z$ (Item (2) of Lemma (4.20). As is easily checked the ideal of $Z$ in $\mathcal{O}_{C_{Z}}$ is locally principal because $[Z] \notin B_{S}$. Thus there is a well-defined residual scheme $Z^{\prime}$ of $Z \beta\left(C_{Z} \cap S\right)$ in $C_{Z}$. The intersection $C_{Z} \cap S$ is a scheme of length 4 by (4.3.8), and hence $\left[Z^{\prime}\right] \in S^{[2]}$. We define the map $\phi_{U}$ of (4.3.9) by setting $\phi_{U}([Z]):=\left(\left[Z^{\prime}\right]\right)$.

Proposition 4.21. Keep notation as above. Then:

(1) $\phi_{U}$ is regular and it extends to a birational involution $\phi: S^{[2]} \cdots>S^{[2]}$,

(2) $H^{2}(\phi)=R_{h}$ where $h=\mu\left(h_{S}\right)-2 \xi_{2}$.

Proof. (1): One verifies easily that $\phi_{U}$ is regular. Furthermore $\phi_{U}(U)=U$ and $\phi_{U}$ is an involution of $U$ : thus $\phi$ is a birational involution. (2): By 4.3.5) and the hypothesis the complement of $U$ in $S^{[2]}$ has codimension 2: since $\phi$ is regular on $U$ it follows that $H^{2}(\phi)$ is locally constant over the family of $K 3$ surfaces given by (4.3.5). Hence arguing as in the proof of Proposition (4.1) we see that it suffices to show that: 
(2a) $\phi^{*} h=h$,

(2b) $\phi^{*} \Delta_{2} \neq \Delta_{2}$,

(2c) $\phi^{*} \sigma^{[2]}=-\sigma^{[2]}$,

where $\sigma^{[2]}$ is the symplectic form induced on $S^{[2]}$ by a symplectic form $\sigma$ on $S$. (2a): Consider the regular map

$$
\begin{array}{ccc}
S^{[2]} & \stackrel{f}{\longrightarrow} & \left|I_{S}(2)\right|^{\vee} \cong \mathbb{P}^{5} \\
{[Z]} & \mapsto & \left\{Q \in\left|I_{S}(2)\right| \mid\langle Z\rangle ß Q\right\} .
\end{array}
$$

One checks easily that $f^{*} c_{1}\left(\mathcal{O}_{\mathbb{P}^{5}}(1)\right)=h$; since $f$ commutes with $\phi$ this proves Item (2a). (2b): If the quadric $\bar{Q}$ of 4.3.5) is chosen generically then the generic conic parametrized by $W(F)$ which is tangent to $\bar{Q}$ intersects $\bar{Q}$ in two other distinct points and hence $\phi^{*} \Delta_{2} \neq \Delta_{2}$. (2c): Let $\left[Z_{i}\right] \in U$ and $\phi\left(\left[Z_{i}\right]\right)=\left[Z_{i}^{\prime}\right]$ for $i=1,2$ : by Lemma (4.20) $W(F)$ is rational and hence

$$
Z_{1}+Z_{1}^{\prime} \sim Z_{2}+Z_{2}^{\prime}
$$

where $\sim$ is rational equivalence. Thus $\phi^{*} \sigma^{[2]}=-\sigma^{[2]}$ by Mumford's Theorem on 0 -cycles (see Prop (22.24) of [34).

Proof of Lemma (4.20). We need to recall Iskovskih's description of a birational map between $F$ and a smooth quadric 3 -fold ((6.5), p.511 of [17]). There exists a line $L_{0} ß F$ such that $N_{L_{0} / F} \cong \mathcal{O}_{L_{0}} \oplus \mathcal{O}_{L_{0}}$. Projection from $L_{0}$ defines a birational map

$$
\pi: F \cdots>Q_{0}
$$

to a smooth quadric 3-fold $Q_{0}$. The indeterminacy locus of $\pi$ is resolved by blowing up $L_{0}$. There is a smooth twisted cubic $Y \beta Q_{0}$ such that the induced regular map $B l_{L_{0}}(F) \rightarrow Q_{0}$ contracts the proper transform of any line in $F$ meeting $L_{0}$ to a point of $Y$. The inverse $\pi^{-1}: Q_{0} \cdots>F$ is given by $\left|I_{Y}(2)\right|$, the linear system of quadrics containing $Y$. The indeterminacy locus of $\pi^{-1}$ is resolved by blowing up $Y$ and the regular map $B l_{Y}\left(Q_{0}\right) \rightarrow F$ contracts the proper transform of every chord of $Y$ contained in $Q_{0}$ (the intersection $\langle Y\rangle \cap Q_{0}$ is a smooth quadric surface). In order to prove (1) of Lemma (4.20) we describe $R(F)$ via $\pi$. Let $[L] \in\left(R(F) \backslash\left\{\left[L_{0}\right]\right\}\right)$. From the above it follows that if $L \cap L_{0}=\emptyset$ then $\pi(L) ß Q_{0}$ is a line meeting $Y$ and not contained in $\langle Y\rangle$, and that viceversa every such line is mapped by $\pi^{-1}$ to a line $L ß F$ with $L \cap L_{0}=\emptyset$. If $L \cap L_{0} \neq \emptyset$ then $\pi$ contracts $L$ to a point and viceversa to every point of $Y$ there corresponds an $[L] \in R(F)$. (Associating to a point $q \in Y$ the unique line $R ß\langle Y\rangle \cap Q_{0}$ such that $R \cap Y=q$ we may view the latter case as a degeneration of the former.) Item (1) of Lemma (4.20) follows easily from the above description of $R(F)$. In order to prove (2) we describe $W(F)$ via $\pi$. Let $[C] \in W(F)$. If $C \cap L_{0}=\emptyset$ then $\pi(C)$ is a conic not contained in $\langle Y\rangle$ with $\pi(C) \cap Y$ a scheme of length 2, and viceversa every conic in $Q_{0}$ not contained in $\langle Y\rangle$ and intersecting $Y$ in a subscheme of length 2 is mapped by $\pi^{-1}$ to a conic $C \beta F$ with $C \cap L_{0}=\emptyset$. If $C \cap L_{0}$ is a point then $\pi$ maps $C$ to a line in $Q_{0}$ not contained in $\langle Y\rangle$, which meets $Y$ if and only if $C$ is reducible, and viceversa every line in $Q_{0}$ and not contained in $\langle Y\rangle$ is mapped by $\pi^{-1}$ to a conic $C \beta F$ such that $C \cap L_{0}$ is a point. (The latter case can be viewed as a degeneration of the former case by adding 
to the line in $Q_{0}$ a suitable chord of $Y$.) Of course if $C \supset L_{0}$ then $f(C)$ is a point of $Y$ and viceversa.... Item (2) of Lemma 4.20) follows easily from the above description of $W(F)$. Let's prove Item (3). By the above description $W(F)$ is irreducible of dimension 4 and the generic conic parametrized by $W(F)$ is smooth. Let $H ß F$ be a smooth hyperplane section: thus $H$ is a del Pezzo surface of degree 5 - the blow up of $\mathbb{P}^{2}$ at 4 points no 3 of which are collinear. Since the set of conics belonging to $H$ has dimension 1 we have a rational map

$$
\begin{array}{ccc}
W(F) & \cdots> & H^{[2]} \\
{[C]} & \mapsto & {[C \cap H] .}
\end{array}
$$

Let $L_{1}, \ldots, L_{10} ß H$ be the 10 lines of $H$ : by Item (2) of Lemma (4.20) the above map is an isomorphism on the complement of $\bigcup_{i=1}^{10} L_{i}^{[2]}$. Thus $W(F)$ is birational to $H^{[2]}$ and hence it is rational.

\subsection{Two involutions}

Let $X$ be an irreducible symplectic variety with $H_{1}, H_{2}$ ample divisors. Let $h_{i}:=c_{1}\left(H_{i}\right)$; we assume that $h_{1}, h_{2}$ are linearly independent. Suppose that there exist regular involutions $\phi_{i}: X \rightarrow X$ for $i=1,2$ such that $H^{2}\left(\phi_{i}\right)=R_{h_{i}}$. Let $\psi:=\phi_{1} \circ \phi_{2}$; then $H^{2}(\psi)$ is described as follows. Let $\mathrm{E}:=\mathbb{R} h_{1} \oplus \mathbb{R} h_{2}$. The restriction of Beauville's form to $\mathrm{L}$ has signature $(1,1)$ and hence we have a direct sum decomposition

$$
H^{2}(X ; \mathbb{R})=\mathrm{E} \oplus \mathrm{L}^{\perp} .
$$

There exist a basis $\left\{e_{+}, e_{-}\right\}$of $\mathrm{L}$ and a real number $l>1$ such that

$$
\left(e_{+}, e_{+}\right)=\left(e_{-}, e_{-}\right)=0, \quad H^{2}(\psi)\left(e_{ \pm}\right)=l^{ \pm 1} .
$$

In particular $\psi$ has infinite order and it should give an interesting dynamical system on $X$ (see the Introduction of [25]). Now assume that $X$ and $\phi_{1}, \phi_{2}$ are defined over a number filed $K$. Following Silverman 31] (now we should assume, and this is always possible, that $h_{i} \in\left(\mathbb{R}_{+} e_{+} \oplus \mathbb{R}_{+} e_{-}\right)$) we may associate to $e_{ \pm}$a normalized logarithmic height $h_{ \pm}$defined on all $p \in X(\bar{K})$ and having the following properties:

(1) $h_{ \pm}(p) \geq 0$ for all $p \in X(\bar{K})$,

(2) $h_{ \pm}(\psi(p))=l^{ \pm 1} h_{ \pm}(p)$,

(3) $h_{+}(p)=h_{-}(p)=0$ if and only if the orbit $\left\{\psi^{j}(p)\right\}_{j \in \mathbb{Z}}$ is finite.

This might be used to produce many rational points on $X$. Silverman's argument shows that there are no $\psi$-invariant effective non-zero cycles of dimension or codimension 1. Thus if $p \in X(\bar{K})$ with $h_{+}(p) \neq 0$ or $h_{-}(p) \neq 0$ then the Zariski-closure of $\left\{\psi^{j}(p)\right\}_{j \in \mathbb{Z}}$ is all of $X$ or some effective cycle of dimension $1<d<(\operatorname{dim} X-1)$. A concrete example: Let $S \beta \mathbb{P}^{3} \times \mathbb{P}^{3}$ be the complete intersection of $\Sigma_{1}, \ldots, \Sigma_{4} \in\left|\mathcal{O}_{\mathbb{P}^{3}}(1) \otimes \mathcal{O}_{\mathbb{P}^{3}}(1)\right|$. For general $\Sigma_{1}, \ldots, \Sigma_{4}$ the surface $S$ is a $K 3$ and the projection $\pi_{i}: S \rightarrow \mathbb{P}^{3}$ to the $i$-th factor is an isomorphism to a smooth quartic with no lines. Let $\ell_{i}:=c_{1}\left(\pi_{i}^{*} \mathcal{O}_{\mathbb{P}^{3}}(1)\right)$. Let $X:=S^{[2]}$ and $h_{i}:=\left(\mu\left(\ell_{i}\right)-\xi_{2}\right)$ where $\mu$ and $\xi_{2}$ are as in (4.1.3); this is an example of the 
situation described above. In fact we have Beauville's involution $\phi_{i}: S^{[2]} \rightarrow S^{[2]}$ with $H^{2}\left(\phi_{i}\right)=R_{h_{i}}$, see Subsection 4.1). Assume that $\Sigma_{1}, \ldots, \Sigma_{4}$ are defined over a number field $K$ : we can show that there exists a finite extension $K^{\prime} \supset K$ with $X\left(K^{\prime}\right)$ Zariski-dense proceeding as follows. There exists a $K^{\prime}$ such that we have a curve $C \in\left|\pi_{1}^{*} \mathcal{O}_{\mathbb{P}^{3}}(1)\right|$ defined over $K^{\prime}$ which is birational to $\mathbb{P}_{K^{\prime}}^{1}$. Then

$$
R:=\left\{[Z] \in S^{[2]} \mid \operatorname{supp}(Z) \in C, \quad[Z] \in \Delta_{2}\right\}
$$

is a ruled surface with $R\left(K^{\prime}\right)$ Zariski-dense in it. One checks that the $\psi$-orbit of the Poincare dual of $R$ is infinite. Since there are no $\psi$-invariant effective non-zero divisors we get that the Zariski closure of $\left\{\psi^{j}\left(R\left(K^{\prime}\right)\right)\right\}_{j \in \mathbb{Z}}$ is the whole of $S^{[2]}$.

\section{Examples: linear systems}

We will give examples of degree-2 polarized deformations of $(K 3)^{[n]}$ which satisfy the hypotheses of Proposition (3.3), i.e. evidence in favor of the L Conjecture (1.2). The examples are inspired by Mukai (Ex.(5.17) [29]): he gave the 4-dimensional example. In proving that the linear systems of our examples are well behaved we will verify that a so-called Strange duality (see [8, 20, 7, 21]) holds for the linear systems in question. We will make the connection with Strange duality in a separate subsection. In the last subsection we will examine more closely the 4-dimensional example.

\subsection{The examples}

Let $S$ be a $K 3$ surface, $D$ an ample divisor on $S$ and let

$$
\mathbf{v}:=2+\ell+2 \eta, \quad\langle\mathbf{v}, \mathbf{v}\rangle \leq 6 .
$$

We assume that both Hypothesis (4.8) and Inequality (4.2.12) hold - recall that this is always possible, see the end of Subsubsection (4.2.2). Thus by Item (1) of Theorem (4.12) the involution $\phi_{\mathbf{v}}: \mathcal{M}(\mathbf{v}) \rightarrow \mathcal{M}(\mathbf{v})$ is regular and by Corollary (4.15) the divisor class $H_{\mathbf{v}}$ is ample. We will show that Proposition (3.3) holds for $X_{0}=\mathcal{M}(\mathbf{v})$ and $H_{0}=H_{\mathbf{v}}$. Let $L$ be the line-bundle on $S$ such that $c_{1}(L)=\ell$ : we assume that

$$
L \text { is ample, } L \cdot L=2 g-2 .
$$

By (5.1.1) we have

$$
\operatorname{dim} \mathcal{M}(\mathbf{v})=2(g-4), \quad g \leq 8 .
$$

It follows from our hypotheses and the results of Mayer 23] that $|L|$ has no base-locus and that the map

$$
f_{S}: S \rightarrow|L|^{\vee} \cong \mathbb{P}^{g}
$$

is an embedding. From now on $S$ is embedded in $\mathbb{P}^{g}$ by the map $f_{S}$. If $g=8$ we make the following extra assumption. Let

$$
\mathbb{G} r\left(2, \mathbb{C}^{6}\right) \hookrightarrow \mathbb{P}\left(\wedge^{2} \mathbb{C}^{6}\right) \cong \mathbb{P}^{14}
$$


be the Plücker embedding: we assume that

$$
S=\mathbb{G} r\left(2, \mathbb{C}^{6}\right) \cap \mathbb{P}^{8}, \quad \mathbb{P}^{8} \text { transversal to } \mathbb{G} r\left(2, \mathbb{C}^{6}\right) .
$$

The generic polarized $K 3$ of degree 14 is obtained in this way, see 28. (This assumption forces us to choose $L, D$ with $L^{\otimes k} \cong \mathcal{O}_{S}(D)$.) Let $\Sigma ß\left|I_{S}(2)\right|$ be the locally closed subset given by

$$
\Sigma:=\left\{Q \in\left|I_{S}(2)\right| \mid \operatorname{rk} Q=6, \quad \operatorname{sing}(Q) \cap S=\emptyset\right\} .
$$

If $Q \in \Sigma$ let $F_{g-3}(Q) \mathbb{B} \mathbb{G} r\left(g-3, \mathbb{P}^{g}\right)$ be the subset parametrizing $(g-3)$ dimensional linear subspaces contained in $Q$; thus $F_{g-3}(Q)$ has two connected components. If $\mathrm{E} \in F_{g-3}(Q)$ the intersection number $(\mathrm{E} \cdot S)_{Q}$ of $\mathrm{E}, S$ as cycles on $Q$ is well-defined because $S$ is contained in the smooth locus of $Q$. If $\mathrm{E}, \mathrm{E}^{\prime} \in$ $F_{g-3}(Q)$ belong to the same connected component then $(\mathrm{E} \cdot S)_{Q}=\left(\mathrm{E}^{\prime} \cdot S\right)_{Q}$. On the other hand if $\mathrm{E}, \mathrm{E}^{\prime}$ belong to different connected components then

$$
(\mathrm{E} \cdot S)_{Q}+\left(\mathrm{E}^{\prime} \cdot S\right)_{Q}=2 g-2 .
$$

Thus if $Q \in \Sigma$ there exists an integer $0 \leq i(Q) \leq g-1$ such that for every $\mathrm{E} \in F_{g-3}(Q)$ we have

$$
(\mathrm{E} \cdot S)_{Q}=(g-1) \pm i(Q)
$$

Let

$$
\Sigma_{a}:=\{Q \in \Sigma \mid \quad i(Q)=a\} .
$$

Each $\Sigma_{a}$ is an open subset of $\Sigma$ and we have $\Sigma=\Sigma_{0} \cup \cdots \cup \Sigma_{g-1}$ (disjoint union). Let $Y:=\bar{\Sigma}_{0}$. Now we are ready to describe $f_{\mathbf{v}}: \mathcal{M}(\mathbf{v}) \cdots>\left|H_{\mathbf{v}}\right|^{\vee}$.

Proposition 5.1. Keep notation and assumptions as above. Then:

(1) $\left|H_{\mathbf{v}}\right|$ has no base-locus.

(2) There is an isomorphism $\left|H_{\mathbf{v}}\right|^{\vee} \cong\left|I_{S}(2)\right|$ such that $\operatorname{Im}\left(f_{\mathbf{v}}\right)=Y$.

(3) The map $f_{\mathbf{v}}: \mathcal{M}(\mathbf{v}) \rightarrow Y$ is finite of degree 2 ; the corresponding covering involution is equal to $\phi_{\mathbf{v}}$.

Since $\left(\mathcal{M}(\mathbf{v}), H_{\mathbf{v}}\right)$ is a degree-2 polarized deformation of $(K 3)^{[n]}$ the above proposition gives examples in favour of the L Conjecture (1.2) for $\operatorname{dim}=4,6,8$. In fact by Corollary (3.3) we get that in each of these dimensions the L Conjecture holds for at least one irreducible component of the relevant moduli space. The proof of the above proposition actually identifies $\mathcal{M}(\mathbf{v})$ with a natural "double cover" of $Y$. We explain this.

Claim 5.2. If $Q \in\left|I_{S}(2)\right|$ then $r k(Q) \geq 5$.

Proof. If $\operatorname{rk}(Q) \leq 4$ there exists a reducible hyperplane section of $S$, contradicting Hypothesis (4.8).

Let $Q \in Y$ : by the above claim $\operatorname{rk}(Q)=5$ or $\operatorname{rk}(Q)=6$ and hence $F_{g-3}(Q)$ has one or two connected components respectively. $\left(F_{g-3}(Q)\right.$ is defined as above also if $\operatorname{rk}(Q)=5$.) Let $\mathcal{F}_{g-3} \rightarrow Y$ be the map with fiber $F_{g-3}(Q)$ over $Q$ and

$$
\mathcal{F}_{g-3} \longrightarrow W \stackrel{\zeta}{\longrightarrow} Y
$$

be its Stein factorization, thus $\zeta^{-1}(Q)$ is the set of connected components of $F_{g-3}(Q)$ : then $\zeta: W \rightarrow Y$ is finite of degree two. Let $\rho: W^{\nu} \rightarrow W$ be the normalization map. 
Proposition 5.3. Keep notation and assumptions as above. The map $f_{\mathbf{v}}: \mathcal{M}(\mathbf{v}) \rightarrow$ $Y$ lifts to a (regular) map $\tilde{f}_{\mathbf{v}}: \mathcal{M}(\mathbf{v}) \rightarrow W$. The map $\mathcal{M}(\mathbf{v}) \rightarrow W^{\nu}$ induced by $\tilde{f}_{\mathbf{v}}$ is an isomorphism.

\subsection{Proof of (5.1)-(5.3)}

\subsubsection{Sheaves on $S$ and quadrics in $\left|I_{S}(2)\right|$}

Let $F$ be a $D$-slope-stable sheaf on $S$ with

$$
v(F)=2+\ell+s \eta .
$$

Let $A ß H^{0}(F)$ be a subspace. Let

$$
\epsilon_{A}: \wedge^{2} A \rightarrow H^{0}\left(\wedge^{2} F\right) \cong H^{0}\left(\mathcal{O}_{S}(1)\right)
$$

be the natural map. Now assume that $\operatorname{dim} A \geq 3$. Let $F_{A} ß F$ be the sheaf generated by $A$. By Lemma (3.5) of [22] the quotient $F / F_{A}$ is Artinian; let $\varnothing_{A}:=\operatorname{supp}\left(F / F_{A}\right) \cup \operatorname{sing}(F)$. Thus $\varnothing_{A}$ is a finite set of points. Let $E_{A}$ be the locally-free sheaf on $S$ fitting into the exact sequence

$$
0 \rightarrow E_{A} \rightarrow A \otimes \mathcal{O}_{S} \rightarrow F_{A} \rightarrow 0 .
$$

We have a regular map

$$
\begin{aligned}
& S \backslash \varnothing_{A} \stackrel{l_{A}}{\longrightarrow} \mathbb{G} r\left(2, A^{\vee}\right) B \mathbb{P}\left(\wedge^{2} A^{\vee}\right) \\
& x \quad \mapsto \operatorname{Ann}\left(E_{A}\right)_{x}
\end{aligned}
$$

Now suppose that $\operatorname{dim} A=4$ : since the Grassmannian above is a quadric hypersurface we get by "pull-back" a quadric in $\left|I_{S}(2)\right|$. To be precise choose a trivialization $\wedge^{4} A^{\vee} \stackrel{\sim}{\rightarrow} \mathbb{C}$ and let $R_{A} \in S y m^{2}\left(\wedge^{2} A\right)$ correspond to multiplication on $\wedge^{2} A^{\vee}$. Let $P_{A}:=\operatorname{Sym}^{2}\left(\epsilon_{A}\right)\left(R_{A}\right)$ : since $R_{A}$ vanishes on $\mathbb{G r} r\left(2, A^{\vee}\right)$ we have

$$
P_{A} \in \operatorname{Ker}\left(\operatorname{Sym}^{2} H^{0}\left(\mathcal{O}_{S}(1)\right) \rightarrow H^{0}\left(\mathcal{O}_{S}(2)\right)\right) .
$$

Lemma 5.4. Keep notation and assumptions as above, in particular assume that $\operatorname{dim} A=4$. Then $P_{A} \neq 0$.

Proof. We claim that

$$
\operatorname{dim}\left(\operatorname{Ker} \epsilon_{A}\right) \leq 1
$$

The lemma follows from the above inequality because $R_{A}$ is non-degenerate. Let $\sigma, \tau \in A$ : we claim that

$$
\text { if } \epsilon_{A}(\sigma \wedge \tau)=0 \text { then } \sigma \wedge \tau=0 .
$$

If $\sigma=0$ there is nothing to prove so we may assume that $\sigma \neq 0$. By $D$ slope-stability of $F$ and Hypothesis (4.8) $\sigma$ defines a map $\sigma: \mathcal{O}_{S} \rightarrow F$ which is injective on fibres away from a finite set $Z ß S$; thus away from $Z$ we have $\tau=f \sigma$ for a regular function $f$. Since $\operatorname{cod}(Z, S)=2$ the function $f$ extends to a regular function on $S$ and hence is equal to a constant $c$; since $F$ is torsion-free we get that $\tau=c \sigma$. Now (5.2.6) gives that $\mathbb{P}\left(\operatorname{Ker} \epsilon_{A}\right) \cap \mathbb{G} r(2, A)=\emptyset$; since $\mathbb{G} r(2, A)$ is a hypersurface in $\mathbb{P}\left(\wedge^{2} A\right)$ this implies (5.2.5).

Definition 5.5. Keep notation and assumptions as above and suppose that $\operatorname{dim} A=4$. Then $Q_{A}:=V\left(P_{A}\right)$ is a well-defined quadric hypersurface in $\mathbb{P}^{g}$ by Lemma 5.4). Clearly $Q_{A} \in\left|I_{S}(2)\right|$ and $r k\left(Q_{A}\right) \leq 6$. When $h^{0}(F)=4$ we set $Q_{F}:=Q_{H^{0}(F)}$. 


\subsubsection{The map $q_{\mathbf{v}}: \mathcal{M}(\mathbf{v}) \rightarrow Y$ and its lift to $W$}

Let $[F] \in \mathcal{M}(\mathbf{v})$. By our hypotheses and Lemma (4.11) we know that $h^{0}(F)=4$. Thus we have a map

$$
\begin{array}{ccc}
\mathcal{M}(\mathbf{v}) & \stackrel{q_{\mathbf{v}}}{\longrightarrow} & \left|I_{S}(2)\right| \\
{[F]} & \mapsto & Q_{F} .
\end{array}
$$

A moment's thought shows that $q_{\mathbf{v}}$ is regular.

Lemma 5.6. Keeping notation as above, $\operatorname{Im}\left(q_{\mathbf{v}}\right)=Y$.

Proof. First we prove that

$$
\operatorname{Im}\left(q_{\mathbf{v}}\right) B Y .
$$

Let $U^{b}(\mathbf{v}) B \mathcal{M}(\mathbf{v})$ be the open subset defined in 4.2.20). Let

$$
[F] \in U^{b}(\mathbf{v}), \quad \phi_{\mathbf{v}}([F]) \neq[F] .
$$

We will show that $Q_{F} \in \Sigma_{0}$. This will prove (5.2.8): in fact $U^{\mathrm{b}}(\mathbf{v})$ is dense in $\mathcal{M}(\mathbf{v})$ by Lemma (4.13), and $\phi_{\mathbf{v}}$ is not the identity (it is anti-symplectic!), hence the set of $[F]$ satisfying (5.2.9) is Zariski-dense in $\mathcal{M}(\mathbf{v})$. Let $\Gamma:=H^{0}(F)$; since $[F] \in U^{\mathrm{b}}(\mathbf{v})$ we have $F_{\Gamma}=F$. By Theorem (4.12) we have $\phi_{\mathbf{v}}([F])=$ $\left[E_{\Gamma}^{\vee}\right]$. Let's show that $\operatorname{rk} Q_{F}=6$, i.e. that $\operatorname{Ker}\left(\epsilon_{\Gamma}\right)=0$. Assume the contrary and let $\alpha \in \operatorname{Ker}\left(\epsilon_{\Gamma}\right)$ be non-zero. Let $K$ be the sheaf on $S$ given by $K:=$ $\operatorname{Ker}\left(\wedge^{2} \Gamma \otimes \mathcal{O}_{S} \rightarrow \wedge^{2} F\right)$ : since $\epsilon_{\Gamma}(\alpha)=0$ we have $\alpha \in H^{0}(K)$. We have a natural exact sequence

$$
0 \rightarrow \wedge^{2} E_{\Gamma} \rightarrow K \stackrel{\pi}{\rightarrow} E_{\Gamma} \otimes F \rightarrow 0 .
$$

By (5.2.6) we know that $\alpha$ is a rank- 4 element of $\wedge^{2} \Gamma$ and hence

$$
\pi(\alpha) \in H^{0}\left(E_{\Gamma} \otimes F\right)=\operatorname{Hom}\left(E_{\Gamma}^{\vee}, F\right)
$$

is an isomorphism. Since $\phi_{\mathbf{v}}([F])=\left[E_{\Gamma}^{\vee}\right]$ this contradicts (5.2.9): thus $\operatorname{rk} Q_{F}=$ 6. Let's show that $\operatorname{sing}\left(Q_{F}\right) \cap S=\emptyset$. The restriction to $S B \mathbb{P}^{g}$ of projection from $\operatorname{sing}\left(Q_{F}\right)$ is identified with $l_{\Gamma}$. If $\operatorname{sing}\left(Q_{F}\right) \cap S \neq \emptyset$ then $\operatorname{sing}\left(Q_{F}\right) \cap S$ is 0 -dimensional by Hypothesis (4.8) and hence $l_{\Gamma}$ is not regular; since $[F] \in U^{\mathrm{b}}(\mathbf{v})$ we have $\emptyset_{\Gamma}=\emptyset$ hence $l_{\Gamma}$ is defined on all of $S$, contradiction. Thus $Q_{F} \in \Sigma$. Let's show that $Q_{F} \in \Sigma_{0}$. Choose a non-zero $\sigma \in H^{0}(F)$ and let

$$
\Gamma_{\sigma}:=\left\{V \in \mathbb{G r} r\left(2, H^{0}(F)^{\vee}\right) \mid f(\sigma)=0, \forall f \in V\right\}
$$

be the corresponding Schubert cycle. There exists a unique $\mathrm{L}_{\sigma} \in F_{g-3}\left(Q_{F}\right)$ which gives $\Gamma_{\sigma}$ when projected from $\operatorname{sing}\left(Q_{F}\right)$. We have $\left(\mathrm{E}_{\sigma} \cdot S\right)_{Q_{F}}=\operatorname{deg}(\sigma)$ where $(\sigma)$ is the zero-locus of $\sigma$ - notice that $(\sigma)$ is 0-dimensional by Hypothesis (4.8). It follows from (5.1.1)-(5.1.2) that $c_{2}(F)=(g-1) \eta$ and hence $\operatorname{deg}(\sigma)=(g-1)$. This shows that $Q_{F} \in \Sigma_{0}$ and thus proves (5.2.8). Now let's prove that $Y \operatorname{Bm}\left(q_{\mathbf{v}}\right)$. Let $Q \in \Sigma^{0}$ : we will show that there exists $[F] \in \mathcal{M}(\mathbf{v})$ such that $Q_{F}=Q$. Projecting $S$ from $\operatorname{sing}(Q) \cong \mathbb{P}^{g-6}$ we get a morphism $l: S \rightarrow \mathbb{P}^{5}$ with $l(S) ß \bar{Q}$, where $\bar{Q} \beta \mathbb{P}^{5}$ is the image of $Q$, a smooth quadric hypersurface. Choose an isomorphism $\bar{Q} \cong \mathbb{G} r\left(2, \mathbb{C}^{4}\right)$ and let $\xi$ be the tautological bundle on $\mathbb{G r} r\left(2, \mathbb{C}^{4}\right)$. Then $F:=l^{*} \xi^{\vee}$ is a vector-bundle on $S$ with

$$
\operatorname{rk}(F)=2, \quad c_{1}(F)=\ell, \quad c_{2}(F)=(g-1) \eta .
$$


(The last equality holds because $Q \in \Sigma_{0}$.) Since $F$ is globally generated and since $h^{2}(F)=0$ (this is easily checked) we get by Lemma (3.5) of $22((3) \Longrightarrow$ $(2))$ that $F$ is $D$-slope-stable. Thus $[F] \in \mathcal{M}(\mathbf{v})$. Since $S ß \mathbb{P}^{g}$ is non-degenerate so is $S ß \mathbb{P}^{5}$ : it follows that $Q_{F}=Q$. This proves that $\Sigma_{0} ß \operatorname{Im}\left(q_{\mathbf{v}}\right)$ : $\operatorname{since} \operatorname{Im}\left(q_{\mathbf{v}}\right)$ is closed we get that $Y \beta \operatorname{Im}\left(q_{\mathbf{v}}\right)$.

The map $q_{\mathbf{v}}$ lifts to a map $\tilde{q}_{\mathbf{v}}: \mathcal{M}(\mathbf{v}) \rightarrow W$ almost by construction. Given $[F] \in \mathcal{M}(\mathbf{v})$ we can associate to $F$ not only the quadric $Q_{F}$ but also a choice of component of $F_{g-3}\left(Q_{F}\right)$ : if $\operatorname{rk}\left(Q_{F}\right)=6$ then $F_{g-3}\left(Q_{F}\right)$ is naturally isomorphic to the variety parametrizing planes in $\mathbb{G} r\left(2, H^{0}(F)^{\vee}\right)$ and hence it is clear how to choose a component of $F_{g-3}\left(Q_{F}\right)$, if $\operatorname{rk}\left(Q_{F}\right)=5$ there is only one component to choose. As is easily checked $\tilde{q}_{\mathbf{v}}$ is regular. Let $\tilde{q}_{\mathbf{v}}^{\nu}: \mathcal{M}(\mathbf{v}) \rightarrow W^{\nu}$ be the (regular) map induced by $\tilde{q}_{\mathbf{v}}$ (recall that $\rho: W^{\nu} \rightarrow W$ is the normalization map).

Lemma 5.7. Keep notation as above. Then $\tilde{q}_{\mathbf{v}}^{\nu}$ is an isomorphism, and hence $q_{\mathbf{v}}: \mathcal{M}(\mathbf{v}) \rightarrow Y$ has degree 2 . Via $\tilde{q}_{\mathbf{v}}^{\nu}$ the involution of $W^{\nu}$ defined by the degree-2 finite map $W^{\nu} \rightarrow Y$ coincides with $\phi_{\mathbf{v}}$.

Proof. We prove the first statement. Clearly $\tilde{q}_{\mathbf{v}}^{\nu}$ is an isomorphism over $\rho^{-1}\left(\Sigma_{0}\right)$. Hence it suffices to verify that $\tilde{q}_{\mathbf{v}}^{\nu}$ has finite fibers. Let $H$ be a hyperplane section of the projective space $\left|I_{S}(2)\right|$ : since $q_{\mathbf{v}} \circ \phi_{\mathbf{v}}=q_{\mathbf{v}}$ we have $\phi_{\mathbf{v}}^{*}\left(q_{\mathbf{v}}^{*} H\right)=q_{\mathbf{v}}^{*} H$. Thus by Proposition (4.14) we have $q_{\mathbf{v}}^{*} H=k H_{\mathbf{v}}$ for some $k \in \mathbb{Z}$. Since $\operatorname{dim} Y \neq 0$ (in fact $\operatorname{dim} Y=\operatorname{dim} \mathcal{M}(\mathbf{v}))$ we get $k \neq 0$, and since $H_{\mathbf{v}}$ is ample by Corollary (4.15) we have

$$
q_{\mathbf{v}}^{*} H \sim k H_{\mathbf{v}}, \quad k>0 .
$$

If $\tilde{q}_{\mathbf{v}}^{\nu}$ has a positive dimensional fiber then $q_{\mathbf{v}}^{*} H$ is trivial on such a fiber: by the above equality also $H_{\mathbf{v}}$ is trivial on that fiber, contradicting ampleness of $H_{\mathbf{v}}$. Let's prove the second statement of the lemma. Let $\iota_{\mathbf{v}}$ be the involution of $W^{\nu}$ defined by the degree-2 finite map $W^{\nu} \rightarrow Y$ : over $\rho^{-1}\left(\Sigma_{0}\right)$ we clearly have $\iota_{\mathbf{v}}=\tilde{q}_{\mathbf{v}}^{\nu} \circ \phi_{\mathbf{v}} \circ\left(\tilde{q}_{\mathbf{v}}^{\nu}\right)^{-1}$, and hence the same equality holds on all of $W^{\nu}$.

\subsubsection{An isomorphism $\left.\left|H_{\mathbf{v}}\right| \stackrel{\cong}{\cong} I_{S}(2)\right|^{\vee}$}

Lemma 5.8. Keep notation and assumptions as above. Then $q_{\mathbf{v}}^{*} H \sim H_{\mathbf{v}}$.

Proof. If $\operatorname{dim} \mathcal{M}(\mathbf{v})=0$, i.e. $g=4$, there is nothing to prove. If $\operatorname{dim} \mathcal{M}(\mathbf{v})=2$, i.e. $g=5$, the result is immediate. In fact in this case $Y=\left|I_{S}(2)\right| \cong \mathbb{P}^{2}$ and hence $\operatorname{deg}\left(q_{\mathbf{v}}^{*} H \cdot q_{\mathbf{v}}^{*} H\right)=\operatorname{deg}\left(q_{\mathbf{v}}\right)=2$; since $\operatorname{deg}\left(H_{\mathbf{v}} \cdot H_{\mathbf{v}}\right)=2$ the result follows from (5.2.10). Assume that $\operatorname{dim} \mathcal{M}(\mathbf{v}) \geq 4$, i.e. that $g \geq 6$. We will prove the lemma by computing the intersection numbers $\operatorname{deg}\left(q_{\mathbf{v}}^{*} H \cdot R\right)$ and $\operatorname{deg}\left(H_{\mathbf{v}} \cdot R\right)$ where $R$ is a certain curve in $\mathcal{M}(\mathbf{v})$ parametrizing singular sheaves. First we construct the curve. Let

$$
\mathbf{w}:=2+\ell+3 \eta \text {. }
$$

Since $g \geq 6$ we have $\mathcal{M}(\mathbf{w}) \neq \emptyset$ by Theorem 4.5). There exists $[V] \in \mathcal{M}(\mathbf{w})$ such that

$$
V \text { is locally-free globally generated, } h^{0}(V)=\chi(V)=5,
$$

see the proof of Lemma (4.17). Choose $p \in S$ and let $R:=\mathbb{P}\left(V_{p}^{\vee}\right)$. Let $\pi_{S}: S \times R \rightarrow S$ be the projection and $\iota: R \hookrightarrow S \times R$ be the inclusion $\iota(x):=$ 
$(p, x)$. Let $\mathcal{F}$ be the sheaf on $S \times R$ fitting into the exact sequence

$$
0 \rightarrow \mathcal{F} \longrightarrow \pi_{S}^{*} V \stackrel{\alpha}{\longrightarrow} \iota_{*} \mathcal{O}_{R}(1) \rightarrow 0
$$

where $\alpha$ is induced by the tautological quotient $V_{p} \otimes \mathcal{O}_{R} \rightarrow \mathcal{O}_{R}(1)$. For $x \in R$ let $F_{x}:=\left.\mathcal{F}\right|_{S \times\{x\}}$. Then $F_{x}$ is torsion-free, $v\left(F_{x}\right)=\mathbf{v}$, and $F_{x}$ is $D$-slope-stable because $V$ is $D$-slope-stable. Since $F_{x} \cong F_{x^{\prime}}$ only if $x=x^{\prime}$ the $R$-flat family $\mathcal{F}$ induces an inclusion $R \hookrightarrow \mathcal{M}(\mathbf{v})$. Let's prove that

$$
\operatorname{deg}\left(H_{\mathbf{v}} \cdot R\right)=1 .
$$

Since $c_{1}\left(H_{\mathbf{v}}\right)=\theta_{\mathbf{v}}(\eta-1)$ we have

$$
\operatorname{deg}\left(H_{\mathbf{v}} \cdot R\right)=\operatorname{deg} \theta_{\mathcal{F}}(\eta-1)=-\int_{S \times R} c h_{3}(\mathcal{F})=\int_{S \times R} c h_{3}\left(\iota_{*} \mathcal{O}_{R}(1)\right) .
$$

Applying Grothendieck-Riemann-Roch one gets that the last term equals 1; this proves (5.2.14). Now let's prove that

$$
\operatorname{deg}\left(q_{\mathbf{v}}^{*} H \cdot R\right)=1 .
$$

We must describe the restriction of $q_{\mathbf{v}}$ to $R$. Let

$$
\begin{array}{ccc}
\mathbb{P}\left(H^{0}(V)^{\vee}\right) & \stackrel{\beta_{V}}{\hookrightarrow}\left|I_{S}(2)\right| \\
x & \mapsto & Q_{A n n(x)}
\end{array}
$$

where $Q_{A n n(x)}$ is as in Definition (5.5) - this makes sense because since $h^{0}(V)=$ 5 we have $\operatorname{dim} \operatorname{Ann}(x)=4$. Let $\Gamma:=H^{0}(V)$ and let $l_{\Gamma}$ be as in (5.2.4). Then $V_{p}^{\vee}$ is naturally identified with $l_{\Gamma}(p)$ and hence

$$
R=\mathbb{P}\left(V_{p}^{\vee}\right)=\mathbb{P}\left(l_{\Gamma}(p)\right) \mathbb{B}\left(H^{0}(V)^{\vee}\right) .
$$

Let $x \in R$ : it is immediate that

$$
q_{\mathbf{v}}\left(\left[F_{x}\right]\right)=\beta_{V}(x) .
$$

Since $\beta_{V}$ is linear Equation (5.2.16) follows. The lemma follows from (5.2.10), (5.2.14) and (5.2.16).

Since $S$ is projectively normal Hirzebruch-Riemann-Roch gives that

$$
\operatorname{dim}\left|I_{S}(2)\right|=d(g):=\frac{1}{2}(g-2)(g-3)-1 .
$$

Lemma 5.9. Keep notation and assumptions as above. Then $Y$ is a nondegenerate subvariety of $\left|I_{S}(2)\right|$.

Proof. If $\operatorname{dim} \mathcal{M}(\mathbf{v})=0$, i.e. $g=4$, then $\left|I_{S}(2)\right|$ is a point and the result is trivially true. If $\operatorname{dim} \mathcal{M}(\mathbf{v})=2$ i.e. $g=5$ then $Y=\left|I_{S}(2)\right|$ and again the result holds. If $\operatorname{dim} \mathcal{M}(\mathbf{v})=4$ i.e. $g=6$ then $\operatorname{dim}\left|I_{S}(2)\right|=5$ and of course $\operatorname{dim} Y=4$. Thus if $Y$ is degenerate it is a hyperplane, and since $\operatorname{deg} q_{\mathbf{v}}=2$ we get by Lemma (5.8) that $\int_{\mathcal{M}(\mathbf{v})} h_{\mathbf{v}}^{4}=2$. On the other hand Fujiki's formula (1.0.1) and the value of Fujiki's constant for deformations of $(K 3)^{[2]}$ (see (4.1.4) $)$ give that 
$\int_{\mathcal{M}(\mathbf{v})} h_{\mathbf{v}}^{4}=12$, contradiction. Now assume that $\operatorname{dim} \mathcal{M}(\mathbf{v})=6$ i.e. $g=7$. Let $\mathbf{w}$ be as in (5.2.11) and $[V] \in \mathcal{M}(\mathbf{w})$ satisfying (5.2.12). Let $\Gamma:=H^{0}(V)$ and

$$
\Delta_{V}:=\bigcup_{p \in S} \mathbb{P}\left(l_{\Gamma}(p)\right) \operatorname{BP}\left(H^{0}(V)^{\vee}\right),
$$

where $l_{\Gamma}$ is as in (5.2.4); thus $\Delta_{V}$ is the image of $\mathbb{P}\left(V^{\vee}\right)$ under the natural map $\zeta_{V}: \mathbb{P}\left(V^{\vee}\right) \rightarrow \mathbb{P}\left(H^{0}(V)^{\vee}\right)$. Proceeding exactly as in the proof of Lemma (5.8) we can associate to each $x \in \mathbb{P}\left(V^{\vee}\right)$ a $D$-slope-stable torsion-free singular sheaf $F_{x}$ on $S$ with $v\left(F_{x}\right)=\mathbf{v}$ : this gives an inclusion $\mathbb{P}\left(V^{\vee}\right) B \mathcal{M}(\mathbf{v})$. By (5.2.18) we have

$$
\left.q_{\mathbf{v}}\right|_{\mathbb{P}\left(V^{\vee}\right)}=\beta_{V} \circ \zeta_{V} .
$$

Since $\Delta_{V}$ spans $\mathbb{P}\left(H^{0}(V)^{\vee}\right)$ and since $\beta_{V}$ is linear we get that

$$
\langle Y\rangle=\left\langle\operatorname{Im}\left(q_{\mathbf{v}}\right)\right\rangle \supset \operatorname{Im}\left(\beta_{V}\right)
$$

where $\langle Y\rangle$ is the span of $Y$. Choose $\left[V^{\prime}\right] \in \mathcal{M}(\mathbf{w})$ with $\left[V^{\prime}\right] \neq[V]$ and $V^{\prime}$ locally-free globally generated with $h^{0}\left(V^{\prime}\right)=\chi\left(V^{\prime}\right)=5$. Thus

$$
\langle Y\rangle \supset \operatorname{Im}\left(\beta_{V}\right) \cup \operatorname{Im}\left(\beta_{V}^{\prime}\right) .
$$

We claim that $\operatorname{Im}\left(\beta_{V}\right)$ and $\operatorname{Im}\left(\beta_{V^{\prime}}\right)$ are disjoint linear spaces. The lemma for $g=7$ follows from this because in this case $\operatorname{dim}\left|I_{S}(2)\right|=9$ by (5.2.19) and on the other hand from (5.2.22) we get that $\operatorname{dim}\langle Y\rangle \geq 9$. Let's prove that $\operatorname{Im}\left(\beta_{V}\right)$ and $\operatorname{Im}\left(\beta_{V^{\prime}}\right)$ are disjoint. Assume that there exists a quadric $Q \in \operatorname{Im}\left(\beta_{V}\right) \cap \operatorname{Im}\left(\beta_{V^{\prime}}\right)$. Projection in $\mathbb{P}^{g}$ with center $\operatorname{sing}(Q)$ defines a rational map $\rho: S \cdots>\mathbb{P}^{k}$ where $k=5$ if $\operatorname{rk}(Q)=6$ and $k=4$ if $\operatorname{rk}(Q)=5$. In the former case $\operatorname{Im}(\rho)$ is contained in a smooth quadric hypersurface which we identify with $\mathbb{G} r\left(2, \mathbb{C}^{4}\right)$ : let $\xi$ be the tautological vector-bundle on $\mathbb{G r} r\left(2, \mathbb{C}^{4}\right)$. Let $J:=S \cap \operatorname{sing}(Q)$; thus $J$ is 0 dimensional by Hypothesis (4.8). By definition of $\beta_{V}, \beta_{V^{\prime}}$ the restriction of $\xi^{\vee}$ to $(S \backslash J)$ is isomorphic to $\left.V\right|_{S \backslash J}$ and to $\left.V^{\prime}\right|_{S \backslash J}$. Thus $\left.\left.V\right|_{S \backslash J} \cong V^{\prime}\right|_{S \backslash J}$ : since $\operatorname{cod}(J, S)=2$ and since $V, V^{\prime}$ are locally-free the isomorphism extends to all of $S$ and hence $[V]=\left[V^{\prime}\right]$, contradiction. If $\operatorname{rk}(Q)=5$ then $\operatorname{Im}(\rho) \beta \mathbb{P}^{4}$ is contained in a smooth quadric hypersurface $\bar{Q}$; embedding $\bar{Q}$ in $\mathbb{G} r\left(2, \mathbb{C}^{4}\right)$ as a hyperplane section for the Plücker embedding we may proceed as in the previous case and we will again arrive at a contradiction. This finishes the proof of the lemma when $g=7$. Finally assume that $\operatorname{dim} \mathcal{M}(\mathbf{v})=8$ i.e. $g=8$. We will show that the subset of $\mathcal{M}(\mathbf{v})$ parametrizing sheaves $F$ with $F^{\vee \vee} / F$ of length 2 has image in $Y$ spanning all of $\left|I_{S}(2)\right|$. Let $\mathbf{u}:=2+\ell+4 \eta$. Then $\langle\mathbf{u}, \mathbf{u}\rangle=-2$ hence by Theorem (4.5) the moduli space $\mathcal{M}(\mathbf{u})$ consists of a single point $[W]$. Arguing as in the proof of Lemma (4.17) one shows that $W$ is locally-free globally generated and that $h^{0}(W)=\chi(W)=6$. Choose a trivialization of $\wedge^{6} H^{0}(W)^{\vee}$ and let

$$
\begin{array}{ccc}
\wedge^{2} H^{0}(W)^{\vee} & \longrightarrow & \operatorname{Sym}^{2}\left(\wedge^{2} H^{0}(W)\right) \\
y & \mapsto & R_{y}
\end{array}
$$

be defined by $R_{y}(\alpha, \beta):=(y \wedge \alpha \wedge \beta)$ for $\alpha, \beta \in \wedge^{2} H^{0}(W)^{\vee}$. Let $\mathbb{G} r:=$ $\mathbb{G} r\left(2, H^{0}(W)^{\vee}\right)$ and $\mathcal{O}_{\mathbb{G r}}(1)$ be the Plücker line-bundle. Then

$$
R_{y} \in \operatorname{Ker}\left(\operatorname{Sym}^{2} H^{0}\left(\mathcal{O}_{\mathbb{G} r}(1)\right) \rightarrow H^{0}\left(\mathcal{O}_{\mathbb{G} r}(2)\right) .\right.
$$

Let $\Gamma:=H^{0}(W)$ and let $\epsilon_{\Gamma}$ be as in (5.2.2); then

$$
P_{y}:=\operatorname{Sym}^{2}\left(\epsilon_{\Gamma}\right)\left(R_{y}\right) \in \operatorname{Ker}\left(\operatorname{Sym}^{2} H^{0}\left(\mathcal{O}_{S}(1)\right) \rightarrow H^{0}\left(\mathcal{O}_{S}(2)\right) .\right.
$$


Claim 5.10. Keep notation and hypotheses as above. If $P_{y}=0$ then $y=0$.

Proof. Recall that $S$ is given by (5.1.4). Let $\xi$ be the tautological rank-two vector-bundle on $\mathbb{G} r\left(2, \mathbb{C}^{6}\right)$. The vector-bundle $\left.\xi^{\vee}\right|_{S}$ is globally generated and as is easily checked $h^{2}\left(\left.\xi^{\vee}\right|_{S}\right)=0$, thus by Lemma (3.5) of 22 $((3) \Longrightarrow(2))$ we get that $\left.\xi^{\vee}\right|_{S}$ is $D$-slope-stable. An easy computation gives $v\left(\left.\xi^{\vee}\right|_{S}\right)=\mathbf{u}$ and thus $\left.\xi^{\vee}\right|_{S} \cong W$. Hence $V\left(P_{y}\right)=V\left(R_{y}\right) \cap \mathbb{P}^{8}$. Since $\mathbb{P}^{8}$ is transversal to $\mathbb{G} r$ the restriction map

$$
H^{0}\left(I_{\mathbb{G} r}(2)\right) \rightarrow H^{0}\left(I_{S}(2)\right)
$$

is injective: this proves the claim.

Since $\operatorname{dim}\left|\mathcal{O}_{\mathbb{G r} r}(1)\right|^{\vee}=14=\operatorname{dim}\left|I_{S}(2)\right|($ use (5.2.19) $)$ we get an isomorphism

$$
\begin{array}{ccc}
\delta: \quad\left|\mathcal{O}_{\mathbb{G} r}(1)\right|^{\vee}=\mathbb{P}\left(\wedge^{2} H^{0}(W)^{\vee}\right) & \stackrel{\sim}{\longrightarrow} & \left|I_{S}(2)\right| \\
{[y]} & \mapsto & {\left[P_{y}\right]}
\end{array}
$$

Let $\Delta_{W} ß \mathbb{P}\left(H^{0}(W)^{\vee}\right)$ be defined as in (5.2.20). Let $x, x^{\prime} \in \Delta_{W}$ be distinct points, thus $x \in \mathbb{P}\left(l_{\Gamma}(p)\right)=\mathbb{P}\left(W_{p}^{\vee}\right), x^{\prime} \in \mathbb{P}\left(l_{\Gamma}\left(p^{\prime}\right)\right)=\mathbb{P}\left(W_{p^{\prime}}^{\vee}\right)$. Let $F_{x, x^{\prime}}$ be the sheaf on $S$ fitting into the exact sequence

$$
0 \rightarrow F_{x, x^{\prime}} \rightarrow V \stackrel{\pi}{\rightarrow} \mathbb{C}_{p} \oplus \mathbb{C}_{p^{\prime}} \rightarrow 0,
$$

where $\pi$ is determined by $x, x^{\prime}$. Then $F_{x, x^{\prime}}$ is torsion-free $D$-slope-stable and $v\left(F_{x, x^{\prime}}\right)=\mathbf{v}$; thus $\left[F_{x, x^{\prime}}\right] \in \mathcal{M}(\mathbf{v})$. Similarly to (5.2.18) we have that

$$
q_{\mathbf{v}}\left(\left[F_{x, x^{\prime}}\right]\right)=\delta\left(\left\langle x, x^{\prime}\right\rangle\right) .
$$

Here $\left\langle x, x^{\prime}\right\rangle \in \mathbb{G} r \beta\left|\mathcal{O}_{\mathbb{G} r}(1)\right|^{\vee}$. Thus

$$
\langle Y\rangle=\left\langle\operatorname{Im}\left(q_{\mathbf{v}}\right)\right\rangle \supset\left\langle\delta\left(\operatorname{chord}\left(\Delta_{W}\right)\right)\right\rangle,
$$

where $\operatorname{chord}\left(\Delta_{W}\right)$ is the set of chords of $\Delta_{W}$, naturally embedded in $\mathbb{G} r \beta\left|\mathcal{O}_{\mathbb{G} r}(1)\right|^{\vee}$. Since $\Delta_{W}$ is a non-degenerate 3 -fold in $\mathbb{P}\left(H^{0}(W)^{\vee}\right) \cong \mathbb{P}^{5}$ it follows that $\operatorname{chord}\left(\Delta_{W}\right)$ is non-degenerate in $\left|\mathcal{O}_{\mathbb{G} r}(1)\right|^{\vee}$. Since $\delta$ is an isomorphism this proves the lemma when $g=8$.

Proposition 5.11. Keep notation and assumptions as above. The map $q_{\mathbf{v}}: \mathcal{M}(\mathbf{v}) \rightarrow$ $\left|I_{S}(2)\right|$ defines an isomorphism $q_{\mathbf{v}}^{*}:\left|I_{S}(2)\right|^{\vee} \stackrel{\sim}{\rightarrow}\left|H_{\mathbf{v}}\right|$.

Proof. By Lemmas (5.8)-(5.9) we have an injective linear map $q_{\mathbf{v}}^{*}:\left|I_{S}(2)\right|^{\vee} \hookrightarrow$ $\left|H_{\mathbf{v}}\right|$. By Lemma (4.15) the divisor $H_{\mathbf{v}}$ is ample; since $K_{\mathcal{M}(\mathbf{v})} \sim 0$ we have $\operatorname{dim}\left|H_{\mathbf{v}}\right|=\left(\chi\left(\mathcal{O}_{\mathcal{M}(\mathbf{v})}\left(H_{\mathbf{v}}\right)\right)-1\right)$. Let $\langle\mathbf{v}, \mathbf{v}\rangle+2=2 n$; since $\mathcal{M}(\mathbf{v})$ is a deformation of $(K 3)^{[n]}$ one knows, see p.96 of [9], that

$$
\chi\left(\mathcal{O}_{\mathcal{M}(\mathbf{v})}\left(H_{\mathbf{v}}\right)\right)=\left(\begin{array}{c}
n+2 \\
2
\end{array}\right) .
$$

Substituting $n=(g-4)$ and using (5.2.19) we get that $\operatorname{dim}\left|I_{S}(2)\right|^{\vee}=\operatorname{dim}\left|H_{\mathbf{v}}\right|$, hence $q_{\mathbf{v}}^{*}$ is an isomorphism.

\subsubsection{Proof of Propositions (5.1)-(5.3).}

Proposition (5.11) identifies $f_{\mathbf{v}}: \mathcal{M}(\mathbf{v}) \rightarrow\left|H_{\mathbf{v}}\right|^{\vee}$ with $q_{\mathbf{v}}: \mathcal{M}(\mathbf{v}) \rightarrow\left|I_{S}(2)\right|$. Since $q_{\mathbf{v}}$ has no base points and $\operatorname{Im}\left(q_{\mathbf{v}}\right)=Y$ we get Items (1)-(2) of Proposition (5.1). Lemma (5.7) gives Item (3) of Proposition (5.1) and also Proposition (5.3) 


\subsection{Strange duality}

The isomorphism

$$
\left|H_{\mathbf{v}}\right|^{\vee} \cong\left|I_{S}(2)\right|
$$

of Item (2) of Proposition (5.1) can be interpreted as a particular case of a conjectural Strange duality between spaces of sections of determinant line-bundles on moduli spaces of sheaves on curves [8] or surfaces [20, 7, 21]. We we will formulate the strange duality statement and then we will make the connection with (5.3.1). Let $(S, D)$ be a polarized $K 3$. For $i=0,1$ let

$$
\mathbf{v}_{i}:=r_{i}+\ell_{i}+s_{i} \eta \in H^{0}(S ; \mathbb{Z})_{\geq 0} \oplus H_{\mathbf{Z}}^{1,1}(S) \oplus H^{4}(S ; \mathbb{Z})
$$

and let $\mathcal{M}\left(\mathbf{v}_{i}\right)$ be the moduli space of pure sheaves $F$ on $S$ with $v(F)=\mathbf{v}_{i}$ and semi-stable with respect to $D$. We assume that $D$ is $\mathbf{v}_{i}$-generic for $i=0,1$ and that $\left(r_{i}+\ell_{i}\right)$ is indivisible, so that Theorems (4.5)-4.7 apply. Assume furthermore that

$$
\left\langle\mathbf{v}_{i}, \mathbf{v}_{1-i}^{\vee}\right\rangle=0
$$

and that

$$
\begin{array}{r}
\left(r_{0} \ell_{1}+r_{1} \ell_{0}\right) \cdot D>0, \\
\text { or }\left(r_{0} \ell_{1}+r_{1} \ell_{0}\right) \cdot D<0,
\end{array}
$$

Let $\mathcal{L}\left(\mathbf{v}_{1-i}\right)$ be the (holomorphic) line-bundle on $\mathcal{M}\left(\mathbf{v}_{i}\right)$ such that

$$
c_{1}\left(\mathcal{L}\left(\mathbf{v}_{1-i}\right)\right)= \begin{cases}\theta_{\mathbf{v}_{i}}\left(-\mathbf{v}_{1-i}^{\vee}\right) & \text { if (5.3.3) holds } \\ \theta_{\mathbf{v}_{i}}\left(\mathbf{v}_{1-i}^{\vee}\right) & \text { if (5.3.4) holds }\end{cases}
$$

Mimicking the proof of Theorem (2.1) of [7] one gets the following.

Proposition 5.12. Keep notation and assumptions as above. There is a section $\sigma_{\mathbf{v}_{1}, \mathbf{v}_{0}} \in H^{0}\left(\mathcal{M}\left(\mathbf{v}_{0}\right) \times \mathcal{M}\left(\mathbf{v}_{1}\right) ; \mathcal{L}\left(\mathbf{v}_{1}\right) \otimes \mathcal{L}\left(\mathbf{v}_{0}\right)\right)$, canonical up to multiplication by a non-zero scalar, such that

$$
\left(\sigma_{\mathbf{v}_{1}, \mathbf{v}_{0}}\right)=\left\{\left(\left[E_{0}\right],\left[E_{1}\right]\right) \in \mathcal{M}\left(\mathbf{v}_{0}\right) \times \mathcal{M}\left(\mathbf{v}_{1}\right) \mid h^{1}\left(E_{0} \otimes E_{1}\right)>0\right\} .
$$

Proof. We assume that there exists a tautological sheaf $\mathcal{F}\left(\mathbf{v}_{i}\right)$ on $S \times \mathcal{M}\left(\mathbf{v}_{i}\right)$ for $i=0,1$; if this is not the case one works with the tautological sheaf on $S \times Q u o t$ where Quot is a suitable Quot-scheme and then applies a descent argument (see [7]). Let $\rho_{i}: S \times \mathcal{M}\left(\mathbf{v}_{0}\right) \times \mathcal{M}\left(\mathbf{v}_{1}\right) \rightarrow S \times \mathcal{M}\left(\mathbf{v}_{i}\right)$ and $\pi: S \times \mathcal{M}\left(\mathbf{v}_{0}\right) \times$ $\mathcal{M}\left(\mathbf{v}_{1}\right) \rightarrow \mathcal{M}\left(\mathbf{v}_{0}\right) \times \mathcal{M}\left(\mathbf{v}_{1}\right)$ be the projections. We consider the line-bundle $\mathcal{L}$ on $\mathcal{M}\left(\mathbf{v}_{0}\right) \times \mathcal{M}\left(\mathbf{v}_{1}\right)$ defined by

$$
\mathcal{L}:=\operatorname{det} \pi_{!}\left(\rho_{0}^{*} \mathcal{F}\left(\mathbf{v}_{0}\right) \otimes \rho_{1}^{*} \mathcal{F}\left(\mathbf{v}_{1}\right)\right)
$$

Let $\left[F_{i}\right] \in \mathcal{M}\left(\mathbf{v}_{i}\right)$. Applying Grothendieck-Riemann-Roch we get that

$$
c_{1}\left(\left.\mathcal{L}\right|_{\left[F_{0}\right] \times \mathcal{M}\left(\mathbf{v}_{1}\right)}\right)=\theta_{\mathbf{v}_{1}}\left(\mathbf{v}_{0}^{\vee}\right), \quad c_{1}\left(\left.\mathcal{L}\right|_{\mathcal{M}\left(\mathbf{v}_{0}\right) \times\left[F_{1}\right]}\right)=\theta_{\mathbf{v}_{0}}\left(\mathbf{v}_{1}^{\vee}\right)
$$

(Recall that $\mathbf{v}_{i}^{\vee} \in \mathbf{v}_{1-i}^{\perp}$ by (5.3.2).) Since $\mathcal{M}\left(\mathbf{v}_{i}\right)$ has $b_{1}=0$ it follows that

$$
\mathcal{L} \cong \begin{cases}\mathcal{L}\left(\mathbf{v}_{1}\right)^{-1} \otimes \mathcal{L}\left(\mathbf{v}_{0}\right)^{-1} & \text { if (5.3.3) holds } \\ \mathcal{L}\left(\mathbf{v}_{1}\right) \otimes \mathcal{L}\left(\mathbf{v}_{0}\right) & \text { if (5.3.4) holds }\end{cases}
$$


By (5.3.2) we have $\chi\left(F_{0} \otimes F_{1}\right)=0$ and furthermore

$$
\begin{aligned}
& H^{2}\left(F_{0} \otimes F_{1}\right)=0 \text { if (5.3.3) holds, } \\
& H^{0}\left(F_{0} \otimes F_{1}\right)=0 \text { if (5.3.4) holds. }
\end{aligned}
$$

It follows by standard arguments that there exists a canonical section

$$
\sigma \in \begin{cases}H^{0}\left(\mathcal{L}^{-1}\right) & \text { if } 5.3 .3 \text { holds, } \\ H^{0}(\mathcal{L}) & \text { if } 5.3 .4 \text { holds }\end{cases}
$$

such that $\sigma\left(\left[F_{0}\right],\left[F_{1}\right]\right)=0$ if and only if $h^{1}\left(F_{0} \otimes F_{1}\right)>0$.

We may view $\sigma_{\mathbf{v}_{1}, \mathbf{v}_{0}}$ as a map

$$
\sigma_{\mathbf{v}_{1}, \mathbf{v}_{0}}: H^{0}\left(\mathcal{M}\left(\mathbf{v}_{0}\right) ; \mathcal{L}\left(\mathbf{v}_{1}\right)\right)^{\vee} \rightarrow H^{0}\left(\mathcal{M}\left(\mathbf{v}_{1}\right) ; \mathcal{L}\left(\mathbf{v}_{0}\right)\right) .
$$

Statement 5.13. [Strange duality] The map $\sigma_{\mathbf{v}_{1}, \mathbf{v}_{0}}$ of [5.3.8) is an isomorphism.

A comment: by Theorem (4.5) $\mathcal{M}\left(\mathbf{v}_{i}\right)$ is a deformation of $(K 3)^{\left[n_{i}\right]}$, where $2 n_{i}=2+\left\langle\mathbf{v}_{i}, \mathbf{v}_{i}\right\rangle$. By a well-known formula, see p.96 of [9], we have

$$
\chi\left(\mathcal{M}\left(\mathbf{v}_{i}\right) ; \mathcal{L}\left(\mathbf{v}_{1-i}\right)\right)=\left(\begin{array}{c}
\frac{1}{2}\left(c_{1}\left(\mathcal{L}\left(\mathbf{v}_{1-i}\right)\right), c_{1}\left(\mathcal{L}\left(\mathbf{v}_{1-i}\right)\right)\right)+n_{i}+1 \\
n_{i}
\end{array}\right) .
$$

By Theorem (4.7) we know that $\theta_{\mathbf{v}_{i}}$ is an isometry and hence we get that

$$
\chi\left(\mathcal{M}\left(\mathbf{v}_{i}\right) ; \mathcal{L}\left(\mathbf{v}_{1-i}\right)\right)=\left(\begin{array}{c}
n_{i}+n_{1-i} \\
n_{i}
\end{array}\right) .
$$

Hence if $\mathcal{L}\left(\mathbf{v}_{0}\right)$ and $\mathcal{L}\left(\mathbf{v}_{1}\right)$ have no higher cohomology there spaces of global sections have equal dimensions: this is consistent with the Strange duality statement. Now we show that the map (5.3.1) is the projectivization of (5.3.8) for suitable $\mathbf{v}_{0}, \mathbf{v}_{1}$. Let

$$
\mathbf{v}:=r+\ell+r \eta, \quad \mathbf{w}:=1-\eta
$$

and assume that Hypothesis (4.8) and (4.2.12) hold. Letting $\mathbf{v}_{0}:=\mathbf{v}, \mathbf{v}_{1}:=\mathbf{w}$ we see that all of our previous assumptions are verifed; let's spell out the Strange duality statement in this case, in particular for $r=2$. We have

$$
c_{1}(\mathcal{L}(\mathbf{w}))=h_{\mathbf{v}}:=\theta_{\mathbf{v}}(\eta-1) .
$$

We let $H_{\mathbf{v}}$ be a divisor on $\mathcal{M}(\mathbf{v})$ such that $c_{1}\left(H_{\mathbf{v}}\right)=h_{\mathbf{v}}$. On the other hand $\mathcal{M}(\mathbf{w})=S^{[2]}$ and

$$
c_{1}(\mathcal{L}(\mathbf{v}))=\mu(\ell)-r \xi_{2}
$$

where $\mu$ and $\xi_{2}$ are as in Subsubsection (4.1.1). Now set $r=2$, let $L$ be the line-bundle such that $c_{1}(L)=\ell$ and let $L \cdot L=(2 g-2)$. We assume that $L$ is very ample and hence $S \beta \mathbb{P}^{g}$ is non-degenerate with $L \cong \mathcal{O}_{S}(1)$. We claim that there is a canonical identification

$$
\left|c_{1}(\mathcal{L}(\mathbf{v}))\right| \cong\left|I_{S}(2)\right| .
$$


In fact if $\mu(L)$ is the line-bundle on $S^{[2]}$ such that $c_{1}(\mu(L))=\mu(\ell)$ we have a canonical identification $|\mu(L)| \cong\left|\mathcal{O}_{\mathbb{P}^{g}}(2)\right|$ and as is easily verified $\left|c_{1}(\mathcal{L}(\mathbf{v}))\right|=$ $\left|\mu(L)\left(-\Delta_{2}\right)\right|$ is the subsystem $\left|I_{S}(2)\right|$. Furthermore $f_{\mathbf{w}}: S^{[2]} \cdots>\left|I_{S}(2)\right|^{\vee}$ is identified with the regular map

$$
\begin{array}{llc}
S^{[2]} & \stackrel{f_{\mathrm{w}}}{\longrightarrow} & \left|I_{S}(2)\right|^{\vee} \\
{[Z]} & \mapsto & \{Q \mid Q \supset\langle Z\rangle\}
\end{array}
$$

( $S$ contains no lines and is cut out by quadrics because of Hypothesis (4.8), and hence $f_{\mathbf{w}}$ is regular.) Thus statement (5.13) asserts that $\sigma_{\mathbf{v}, \mathbf{w}}$ gives an isomorphism $\left|I_{S}(2)\right|^{\vee} \cong\left|H_{\mathbf{v}}\right|$. Proposition (5.1) gives such an isomorphism for $g \leq 8$ - to be precise when $g=8$ we have the extra assumption (5.1.4). Let's show that Isomorphism (5.3.1) is equal to the projectivization of $\sigma_{\mathbf{v}, \mathbf{w}}$. Let $q_{\mathbf{v}}$ be the map of (5.2.7).

Claim 5.14. Keep notation as above. Let $[F] \in \mathcal{M}(\mathbf{v})$ and assume that $q_{\mathbf{v}}([F]) \in \Sigma_{0}$. Let $[Z] \in S^{[2]}$. Then $q_{\mathbf{v}}([F]) \in f_{\mathbf{w}}([Z])$ if and only if $h^{1}\left(I_{Z} \otimes F\right)>$ 0 .

Proof. By definition $q_{\mathbf{v}}([F]) \in f_{\mathbf{w}}([Z])$ if and only if $Q_{F} \supset\langle Z\rangle$. By the definition of $Q_{F}$ (see Definition (5.5) ) this is equivalent to the existence of a non-zero $\tau \in H^{0}(F)$ vanishing on $Z$, i.e. to $h^{0}\left(I_{Z} \otimes F\right)>0$. Since $\chi\left(I_{Z} \otimes F\right)$ and $h^{2}\left(I_{Z} \otimes F\right)$ both vanish the claim follows.

Now let $\gamma:\left|I_{S}(2)\right| \stackrel{\sim}{\rightarrow}\left|H_{\mathbf{v}}\right|^{\vee}$ be the inverse of the isomorphism of Proposition (5.1) and consider the composition

$$
\gamma \circ \sigma_{\mathbf{w}, \mathbf{v}}:\left|H_{\mathbf{v}}\right|^{\vee} \cdots>\left|H_{\mathbf{v}}\right|^{\vee},
$$

a priori a rational linear map. Let $x \in \Sigma_{0}$ : by Claim (5.14) we know that $\gamma \circ \sigma_{\mathbf{w}, \mathbf{v}}$ is regular at $x$ and that $\gamma \circ \sigma_{\mathbf{w}, \mathbf{v}}(x)=x$. By Lemma (5.9) $\Sigma_{0}$ is non-degenerate and hence $\gamma \circ \sigma_{\mathbf{w}, \mathbf{v}}$ is regular everywhere and equal to the identity.

\subsection{The 4-dimensional case}

Keep notation and assumptions as in the introduction to Subsection (5.1) and suppose that $g=6$. Then by (5.1.3) we have $\operatorname{dim} \mathcal{M}(\mathbf{v})=4$; set $X:=\mathcal{M}(\mathbf{v})$. We will present a couple of observations on $Y=f_{\mathbf{v}}(X)$. We assume that $S$ is the generic $K 3$ of genus $g$ (this forces $\mathcal{O}_{S}(D) \cong L^{\otimes k}$ ) and hence

$$
S=F \cap \bar{Q}
$$

where $F$ is the Fano 3-fold given by (4.3.4) and $\bar{Q}$ is a quadric hypersurface transversal to $F$, see (4.3.5). By (5.2.19) we have $\operatorname{dim}\left|I_{S}(2)\right|=5$. Let $\Sigma$ be the divisor on $\left|I_{S}(2)\right|$ of singular quadrics (i.e. of quadrics of rank at most 6 ). Since $\operatorname{dim}\left|I_{F}(2)\right|=4$ and every quadric containing $F$ is singular we have

$$
\Sigma=\left|I_{F}(2)\right|+\Sigma^{\prime}
$$

where $\Sigma^{\prime}$ is an effective divisor of degree 6 . The hypersurface $Y$ is irreducible and non-degenerate and $Y \beta s u p p(\Sigma)$, hence $Y$ ßsupp $\left(\Sigma^{\prime}\right)$. By Item (3) of Proposition (5.1) we know that $\operatorname{deg}\left(f_{\mathbf{v}}: X \rightarrow Y\right)=2$ and by (4.1.4) we have $\int_{X} f_{\mathbf{v}}^{*} c_{1}\left(\mathcal{O}_{\mathbb{P}^{5}}(1)\right)^{4}=$ 12, thus $\operatorname{deg} Y=6$ : this gives that $\Sigma^{\prime}=Y$ i.e.

$$
\Sigma=\left|I_{F}(2)\right|+Y .
$$




\subsubsection{A closer view of $Y$}

Let $\operatorname{Fix}\left(\phi_{\mathbf{v}}\right) \beta X$ be the locus of fixed points of $\phi_{\mathbf{v}}$; since $\phi_{\mathbf{v}}$ is anti-symplectic Fix $\left(\phi_{\mathbf{v}}\right)$ is a smooth Lagrangian surface. Let $\widehat{X} \rightarrow X$ be the blow up of Fix $\left(\phi_{\mathbf{v}}\right)$ : then $\phi_{\mathbf{v}}$ acts on $\widehat{X}$ with smooth quotient $\widehat{Y}$. Since $\phi_{\mathbf{v}}$ acts trivially on $K_{X}$ the 4 -fold $\widehat{Y}$ is a Calabi-Yau. The natural map $\widehat{Y} \rightarrow X /\left\langle\phi_{\mathbf{v}}\right\rangle$ is a resolution of singularities.

Claim 5.15. $Y$ is isomorphic to $X /\left\langle\phi_{\mathbf{v}}\right\rangle$ and via this isomorphism the map $f_{\mathbf{v}}: X \rightarrow Y$ is identified with the quotient map $X \rightarrow X /\left\langle\phi_{\mathbf{v}}\right\rangle$.

Proof. The map $f_{\mathbf{v}}$ commutes with $\phi_{\mathbf{v}}$ hence it descends to a map $X /\left\langle\phi_{\mathbf{v}}\right\rangle \rightarrow Y$ which is finite of degree 1: we must show that this map is an isomorphism. It suffices to show that $Y$ is normal, and since $Y$ is a hypersurface this is equivalent to $Y$ being smooth in codimension 1. The Calabi-Yau $\widehat{Y}$ is birational to $Y$ and hence any desingularization of $Y$ has Kodaira dimension equal to 0; since $\operatorname{deg} Y=6$ we get by adjunction that $Y$ is smooth in codimension 1 .

It follows from the claim that $\operatorname{sing}(Y)$ is a smooth surface and that at a point $p \in \operatorname{sing}(Y)$ the 4 -fold $Y$ is modelled on $\left(\mathbb{C}^{2} \times V\left(x^{2}+y^{2}+z^{2}\right), 0\right)$. The following result gives a moduli-theoretic interpretation of the intersection $Y \cap\left|I_{F}(2)\right|$.

Claim 5.16. We have $f_{\mathbf{v}}^{*}\left|I_{F}(2)\right|=\Delta(\mathbf{v})+\Theta(\mathbf{v})$ where $\Delta(\mathbf{v}), \Theta(\mathbf{v})$ are given by (4.2.24) and Lemma 4.17) respectively.

Proof. Let $\mathbf{w}$ be as in (5.2.11); since $g=6$ the moduli space $\mathcal{M}(\mathbf{w})$ consists of a single point $[V]$ and $V$ satisfies (5.2.12). Let $\beta_{V}$ be the map of (5.2.17). As is easily checked $\operatorname{Im}\left(\beta_{V}\right)=\left|I_{F}(2)\right|$. Proceeding as in the proof of Lemma (15.9), the case $g=7$ (in the present proof $g=6$ but it makes no difference), we get $\zeta_{V}: \mathbb{P}\left(V^{\vee}\right) \rightarrow \mathbb{P}\left(H^{0}(V)^{\vee}\right)$. For $x \in \mathbb{P}\left(V^{\vee}\right)$ we let $F_{x}$ be the singular sheaf on $S$ defined in the proof of Lemma [5.8); thus $\left[F_{x}\right] \in \Delta(\mathbf{v})$. In fact we have an identification

$$
\begin{array}{ccc}
\mathbb{P}\left(V^{\vee}\right) & \stackrel{\sim}{\longrightarrow} & \Delta(\mathbf{v}) \\
x & \mapsto & {\left[F_{x}\right]}
\end{array}
$$

Proposition (5.1) identifies $f_{\mathbf{v}}$ with $q_{\mathbf{v}}$ and hence (5.2.21) becomes (via Identification (5.4.4) ) the equality $\left.f_{\mathbf{v}}\right|_{\Delta(\mathbf{v})}=\beta_{V} \circ \zeta_{V}$; hence

$$
f_{\mathbf{v}}^{*}\left|I_{F}(2)\right|=\Delta(\mathbf{v})+\phi_{\mathbf{v}}^{*} \Delta(\mathbf{v}) .
$$

By (4.2.26) we have $\phi_{\mathbf{v}}^{*} \Delta(\mathbf{v})=\Theta(\mathbf{v})$.

\subsubsection{The dual of $Y$}

Going back to Strange duality we set $\mathbf{w}:=1-\eta$ as in (5.3.9). Thus we have the map $f_{\mathbf{w}}$ of (5.3.13). Let $Y_{\mathbf{w}}:=\operatorname{Im}\left(f_{\mathbf{w}}\right)$ and $Y_{\mathbf{v}}:=\operatorname{Im}\left(f_{\mathbf{v}}\right)$. Let $Y_{\mathbf{v}}^{\vee} \beta\left|I_{S}(2)\right|^{\vee}$ be the dual of $Y_{\mathbf{v}}$; thus we have a birational map

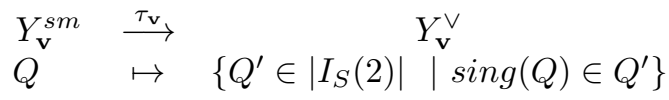

Proposition 5.17. Keep notation as above. Then $Y_{\mathbf{w}}=Y_{\mathbf{v}}^{\vee}$. 
Proof. Let $Q \in f_{\mathbf{v}}\left(U^{\mathrm{b}}(\mathbf{v}) \backslash F i x\left(\phi_{\mathbf{v}}\right)\right)$. Then $\operatorname{rk}(Q)=6$ and if $\{p\}:=\operatorname{sing}(Q)$ we have $p \notin S$. We claim that there exists $[Z] \in S^{[2]}$ such that $p \in\langle Z\rangle$. Assume the contrary. Let $\mathrm{E} \mathbb{P}^{6}$ be a a hyperplane not containing $p$ and $Q_{0}:=Q \cap \mathrm{E}$. Projection from $p$ defines a regular map $\pi: S \rightarrow Q_{0}$ which is is an embedding because no chord of $S$ contains $p$. The exact sequence of vector-bundles

$$
0 \rightarrow T_{S} \rightarrow \pi^{*} T_{Q_{0}} \rightarrow N_{S / Q_{0}} \rightarrow 0
$$

gives that $\int_{S} c_{2}\left(N_{S / Q_{0}}\right)=46$. Let $\left[\pi_{*}(S)\right] \in H^{4}\left(Q_{0}\right)$ be the Poincarè dual of $\pi_{*}(S)$; since $\pi$ is an embedding we have

$$
\int_{Q_{0}}\left[\pi_{*}(S)\right] \wedge\left[\pi_{*}(S)\right]=\int_{S} c_{2}\left(N_{S / Q_{0}}\right)=46 .
$$

On the other hand we see directly that the left-hand side is equal to 50 , contradiction. Thus there exists $[Z] \in S^{[2]}$ such that $p \in\langle Z\rangle$. Clearly $\tau_{\mathbf{v}}(p)=f_{\mathbf{w}}([Z])$. Thus $Y_{\mathbf{v}}^{\vee} \beta Y_{\mathbf{w}}$. Since both are irreducible hypersurfaces we get that $Y_{\mathbf{v}}^{\vee} \beta Y_{\mathbf{w}}$.

Corollary 5.18. Keep notation as above. Then $\operatorname{deg} Y_{\mathbf{w}}=6$ and $f_{\mathbf{w}}$ has degree 2 onto its image.

Proof. The map $f_{\mathbf{w}}$ is base-point free, it commutes with the involution $\phi_{\mathbf{v}}$ and $\int_{S^{[2]}} f_{\mathbf{w}}^{*} c_{1}\left(\mathcal{O}_{\mathbb{P}^{5}}(1)\right)^{4}=12$. Thus $f_{\mathbf{w}}$ is of finite degree $2 d$ over its image $Y_{\mathbf{w}}$ and $\operatorname{deg}\left(Y_{\mathbf{w}}\right)=\frac{6}{d}$. On the other hand by Proposition (5.17) we know that any desingularization of $Y_{\mathbf{w}}$ has Kodaira dimension 0 and hence by adjunction $\operatorname{deg}\left(Y_{\mathbf{w}}\right) \geq 6$. Thus $\operatorname{deg} Y_{\mathbf{w}}=6$ and $d=1$.

\section{Connecting the examples}

Let $\mathbf{v}$ be given by (4.2.10), and assume that Hypothesis (4.8) and (4.2.12) hold. Assume also that

$$
2 n-2:=\langle\mathbf{v}, \mathbf{v}\rangle \leq 4 r-2 .
$$

Let $h_{\mathbf{v}}:=\theta_{\mathbf{v}}(\eta-1)$ and let $H_{\mathbf{v}}$ be a divisor on $\mathcal{M}(\mathbf{v})$ such that $c_{1}\left(H_{\mathbf{v}}\right)=h_{\mathbf{v}}$. Then $\left(\mathcal{M}(\mathbf{v}), H_{\mathbf{v}}\right)$ is a degree-2 polarized deformation of $(K 3)^{[n]}$, see Item $(2)$ of Corollary 4.15) for $r \geq 2$ and Subsubsection 4.1.3 for the case $r=1$. Choosing different v's we get many different families of degree-2 polarized varieties of the same dimension: the methods that prove Theorem (4.5) should also show that these varieties are polarized deformation equivalent, i.e. that they are "parametrized" by the same connected component of $\mathcal{Q}_{n}^{0}$ (see (1.0.4)). In other words we expect that given $\left(\mathcal{M}(\mathbf{v}), H_{\mathbf{v}}\right)$ and $\left(\mathcal{M}(\mathbf{w}), H_{\mathbf{w}}\right)$ as above of the same dimension there exist a proper submersive map of connected complex manifolds $\pi: \mathcal{X} \rightarrow B$, a relatively ample divisor $\mathcal{H}$ on $\mathcal{X}$, and $t, u \in B$ such that $\left(X_{t}, H_{t}\right) \cong\left(\mathcal{M}(\mathbf{v}), H_{\mathbf{v}}\right)$ and $\left(X_{u}, H_{u}\right) \cong\left(\mathcal{M}(\mathbf{w}), H_{\mathbf{w}}\right)$. We will prove that this is indeed the case in one significant example. Let $(S, D)$ be a polarized $K 3$ of degree 10 and let

$$
\mathbf{v}:=2+c_{1}(D)+2 \eta
$$

We assume that Hypothesis (4.8) and (4.2.12) hold with $\ell$ replaced by $c_{1}(D)$ : thus $\mathcal{M}(\mathbf{v})$ (stability is with respect to $D$ ) is a deformation of $(K 3)^{[2]}$ and $H_{\mathbf{v}}$ is 
a degree-2 polarization of $\mathcal{M}(\mathbf{v})$. Let $T ß \mathbb{P}^{3}$ be a quartic surface not containing lines and let $A$ be the (hyper)plane class on $T$. We let

$$
\mathbf{w}:=1+c_{1}(A)+\eta
$$

Then $\mathcal{M}(\mathbf{w})=T^{[2]}$ and $H_{\mathbf{w}}$ is ample of degree two.

Proposition 6.1. Keep notation and assumptions as above. Then $\left(\mathcal{M}(\mathbf{v}), H_{\mathbf{v}}\right)$ is polarized deformation equivalent to $\left(\mathcal{M}(\mathbf{w}), H_{\mathbf{w}}\right)$.

Proof. By surjectivity of the period map for $K 3$ 's there exists a quartic $T_{0} ß \mathbb{P}^{3}$ containing a line $R$ and such that $H_{\mathbb{Z}}^{1,1}\left(T_{0}\right)=\mathbb{Z} c_{1}(R) \oplus \mathbb{Z} c_{1}\left(A_{0}\right)$ where $A_{0}$ is the (hyper)plane class. Let

$$
\mathbf{w}_{0}:=1+c_{1}\left(A_{0}\right)+\eta .
$$

The divisor $D_{0}:=2 A_{0}-R$ is ample of degree 10 thus $\left(T_{0}, D_{0}\right)$ is a degree-10 polarized $K 3$. Let

$$
\mathbf{v}_{0}:=2+c_{1}\left(D_{0}\right)+2 \eta
$$

and let $\mathcal{M}\left(\mathbf{v}_{0}\right)$ be the moduli space with (semi)stability with respect to $D_{0}$. As is easily checked $D_{0}$ is $\mathbf{v}_{0}$-generic and hence by Theorem (4.5) we know that $\mathcal{M}\left(\mathbf{v}_{0}\right)$ is smooth. We will define a birational map

$$
\gamma: \mathcal{M}\left(\mathbf{w}_{0}\right) \cdots>\mathcal{M}\left(\mathbf{v}_{0}\right) .
$$

Let $[Z] \in T_{0}^{[2]}=\mathcal{M}\left(\mathbf{w}_{0}\right)$ : an easy computation gives that

$$
\operatorname{dim} \operatorname{Ext}^{1}\left(I_{Z}\left(A_{0}\right), \mathcal{O}_{T_{0}}\left(A_{0}-R\right)\right)= \begin{cases}1 & \text { if } Z \not \subset R \\ 2 & \text { if } Z \subset R\end{cases}
$$

Furthermore any non-trivial extension

$$
0 \rightarrow \mathcal{O}_{T_{0}}\left(A_{0}-R\right) \rightarrow E \rightarrow I_{Z}\left(A_{0}\right) \rightarrow 0
$$

is $D_{0}$-slope-stable and $v(E)=\mathbf{v}_{0}$. Since for $[Z] \notin R^{(2)}$ we have a non-trivial extension $E_{Z}$ as above unique up to isomorphism, we get a well-defined regular map

$$
\begin{array}{ccc}
\left(\mathcal{M}\left(\mathbf{w}_{0}\right) \backslash R^{(2)}\right) & \longrightarrow & \mathcal{M}\left(\mathbf{v}_{0}\right) \\
{[Z]} & \mapsto & E_{Z} .
\end{array}
$$

Let $[F] \in \mathcal{M}\left(\mathbf{v}_{0}\right)$; then $\chi\left(F\left(R-A_{0}\right)\right)=1$ and since by stability we have $h^{2}\left(F\left(R-A_{0}\right)\right)=0$ we get that $h^{0}\left(F\left(R-A_{0}\right)\right) \geq 1$. One checks easily that 6.0.4) is an isomorphism onto the open dense subset of $\mathcal{M}\left(\mathbf{v}_{0}\right)$ parametrizing sheaves $F$ such that $h^{0}(F)=1$ : we define (6.0.3) to be the birational map that corresponds to (6.0.4). One easily shows that $\gamma$ is the flop (see [27]) of $R^{(2)}$. Furthermore - and this is the main point - we have $\gamma^{*} H_{\mathbf{v}_{0}}=H_{\mathbf{w}_{0}}$. If $\gamma$ were regular we would be done; since $\gamma$ is not regular we proceed as follows. Let $\mathcal{X} \rightarrow B_{\mathbf{w}_{0}}$ be a representative for the deformation space of $\left(\mathcal{M}\left(\mathbf{w}_{0}\right), H_{\mathbf{w}_{0}}\right)$, i.e. deformations of $\mathcal{M}\left(\mathbf{w}_{0}\right)$ that "keep $H_{\mathbf{w}_{0}}$ of type $(1,1)$ ". Similarly let $\mathcal{X}^{\prime} \rightarrow B_{\mathbf{v}_{0}}$ be a representative for the deformation space of $\left(\mathcal{M}\left(\mathbf{v}_{0}\right), H_{\mathbf{v}_{0}}\right)$. Thus there is a divisor $\mathcal{H}$ on $\mathcal{X}$ such that for every $s \in B_{\mathbf{w}_{0}}$ the pull-back of $\mathcal{H}$ to $X_{s}$, call it $H_{s}$, is of degree 2 and of course $H_{0} \sim H_{\mathbf{w}_{0}}$. Similarly we have $\mathcal{H}^{\prime}$ on $\mathcal{X}^{\prime}$. Now let $\mathrm{E}=R^{(2)} \beta T_{0}^{[2]}=\mathcal{M}\left(\mathbf{w}_{0}\right)$ and let $\mathrm{E}^{\prime} \beta \mathcal{M}\left(\mathbf{w}_{0}\right)$ be the corresponding copy of $R^{(2)}$ 
- the indeterminacy locus of $\gamma^{-1}$. Let $B_{\mathbf{w}_{0}}(\mathrm{~L}) ß B_{\mathbf{w}_{0}}$ be the locus parametrizing deformations of $\left(\mathcal{M}\left(\mathbf{w}_{0}\right), H_{\mathbf{w}_{0}}\right)$ for which $\mathrm{E}$ deforms too, and define similarly $B_{\mathbf{v}_{0}}\left(\mathrm{E}^{\prime}\right)$. By a Theorem of Voisin [33] each of these loci is smooth of codimension 1.

Claim 6.2. If we shrink enough $B_{\mathbf{w}_{0}}$ and $B_{\mathbf{v}_{0}}$ around 0 the following holds. Let $s \in\left(B_{\mathbf{w}_{0}} \backslash B_{\mathbf{w}_{0}}\left(E^{\prime}\right)\right)$. There exists $u \in\left(B_{\mathbf{v}_{0}} \backslash B_{\mathbf{v}_{0}}\left(E^{\prime}\right)\right.$ such that $\left(X_{s}, H_{s}\right) \cong$ $\left(X_{u}^{\prime}, H_{u}^{\prime}\right)$.

Proof. Let $\Gamma \beta \mathcal{X}$ be the locus swept out by the $\mathbb{P}^{2}$ 's which are deformations of $\mathrm{E}$ and define similarly $\Gamma^{\prime} \beta \mathcal{X}^{\prime}$; thus we have $\mathbb{P}^{2}$-bundles $\Gamma \rightarrow B_{\mathbf{w}_{0}}(\mathrm{E})$ and $\Gamma^{\prime} \rightarrow B_{\mathbf{w}_{0}}\left(\mathrm{E}^{\prime}\right)$. Let $\pi: \mathcal{Y} \rightarrow \mathcal{X}$ be the blow up of $\Gamma$. Let $E$ be the exceptional divisor of $\pi$; thus $\pi$ gives a $\mathbb{P}^{2}$-bundle $E \rightarrow \Gamma$. Following Huybrechts [13] we see that $E$ has another $\mathbb{P}^{2}$-fibration structure $E \rightarrow \Gamma^{\prime}$ and that one can contract $\mathcal{Y}$ along this fibration and get a smooth $\mathcal{X}^{\prime \prime}$. We still have a map $\mathcal{X}^{\prime \prime} \rightarrow B_{\mathbf{v}_{0}}$ which is submersive, and the divisor $\mathcal{H}^{\prime \prime}$ - the transform of $\mathcal{H}$. If $s \notin B_{\mathbf{w}_{0}}(\mathrm{E})$ then $\left(X_{s}^{\prime \prime}, H_{s}^{\prime \prime}\right) \cong\left(X_{s}, H_{s}\right)$. If $s \in B_{\mathbf{w}_{0}}(\mathrm{E})$ then $X_{s}^{\prime \prime}$ is the flop of $X_{s}$ with center $\mathrm{E}_{s}$ (the deformation of $\mathrm{E}$ ) and $H_{s}^{\prime \prime}$ is the divisor corresponding to $H_{s}$ via the flop; in particular $X_{0}^{\prime \prime} \cong \mathcal{M}\left(\mathbf{v}_{0}\right)$. Considering the period map of $\mathcal{X}^{\prime \prime}$ we get that $\mathcal{X}^{\prime \prime} \rightarrow B_{\mathbf{w}_{0}}$ is the deformation space of $\left(\mathcal{M}\left(\mathbf{v}_{0}\right), H_{\mathbf{v}_{0}}\right)$. The claim follows immediately.

The proposition follows immediately from the above claim. In fact let $s \in$ $B_{\mathbf{w}_{0}}$ parametrize $\left(\mathcal{M}(\mathbf{w}), H_{\mathbf{w}}\right)$ - such an $s$ exists as long as $T$ is sufficiently close to $T_{0}$. Since $T$ does not contain lines $s \notin B_{\mathbf{w}_{0}}(\mathrm{E})$; by the claim there exists $\hat{u} \in B_{\mathbf{v}_{0}}$ such that $\left(\mathcal{M}(\mathbf{w}), H_{\mathbf{w}}\right) \cong\left(X_{\hat{u}}^{\prime}, H_{\hat{u}}^{\prime}\right)$. Since the moduli space of polarized $K 3$ 's of degree 10 (or any other degree) is irreducible $\left(\mathcal{M}(\mathbf{v}), H_{\mathbf{v}}\right)$ is parametrized by a point $\bar{u} \in B_{\mathbf{v}_{0}}$ (again we want $(S, D)$ sufficiently close to $\left.\left(T_{0}, D_{0}\right)\right)$ i.e. $\left(\mathcal{M}(\mathbf{v}), H_{\mathbf{v}}\right) \cong\left(X_{\bar{u}}^{\prime}, H_{u}^{\prime}\right)$. Since the locus of $u \in B_{\mathbf{v}_{0}}$ such that $H_{u}$ is ample is Zariski open we get the proposition.

\section{References}

[1] D. Barlet, Espace analytique réduit des cycles analytiques complexes compacts d'un espace analytique complexe de dimension finie, Lecture Notes in Math., Vol. 482, Springer, 1975, pp. 1-158.

[2] W. Barth, C. Peters, A. Van de Ven, Compact complex surfaces, Ergebnisse der Mathematik und ihrer Grenzgebiete 3. Folge, 4, Springer, 1984.

[3] A. Beauville, Variétes Kähleriennes dont la premiére classe de Chern est nulle, J. Differential geometry 18, 1983, pp. 755-782.

[4] A. Beauville, Some remarks on Kähler manifolds with $c_{1}=0$, Classification of algebraic and analytic manifolds (Katata, 1982), Progr. Math. 39, Birkhäuser, 1983, pp. 1-26.

[5] A. Beauville, R. Donagi, La variétés des droites d'une hypersurface cubique de dimension 4. C. R. Acad. Sci. Paris Sér. I Math. 301, 1985, pp. 703-706.

[6] F. Bogomolov, Hamiltonian Kählerian manifolds, Soviet Math. Dokl. 19 (1978), 1979, pp. 1462-1465. 
[7] G. Danila, Résultats sur la conjecture de dualité étrange sur le plan projectif, Bull. Soc. math. France 130, 2002, pp. 1-33.

[8] R. Donagi, L. W. Tu, Theta functions for $S L(n)$ versus $G L(n)$, Math. Res. Letters 1, 1994, pp. 345-357.

[9] G. Ellingsrud, L. Göttsche, M. Lehn, On the cobordism class of the Hilbert scheme of a surface, J. Algebraic Geometry 10, 2001, pp. 81-100.

[10] A. Fujiki, On the de Rham Cohomology Group of a Compact Kähler Symplectic Manifold, Adv. Studies in Pure Math. 10, Algebraic Geometry, Sendai 1985, pp. 105-165, 1987.

[11] W. Fulton, Intersection theory, Ergebnisse der Mathematik und ihrer Grenzgebiete (3), 2. Springer-Verlag, Berlin, 1984

[12] D. Gieseker, On the moduli of vector bundles on an algebraic surface, Ann. of Math. 106, 1977, pp. 45-60.

[13] D. Huybrechts, Birational symplectic manifolds and their deformations, J. Differential Geom. 45, 1997, pp. 488-513.

[14] D. Huybrechts, Compact hyper-Khler manifolds: basic results, Invent. Math. 135, 1999, pp. 63-113.

[15] D. Huybrechts, Erratum: "Compact hyper-Khler manifolds: basic results"[Invent. Math. 135 (1999), no. 1, 63-113], Invent. Math. 152, 2003, pp. 209-212.

[16] D. Huybrechts, M. Lehn The geometry of moduli spaces of sheaves, Aspects of Mathematics vol. E31, Vieweg 1997.

[17] V. A. Iskovskih, Fano 3-folds, I, Math. USSR Izvestija, Vol. 11, 1977, pp. 485527.

[18] Y. Kawamata, K. Matsuda, K. Matsuki, Introduction to the Minimal Model Problem, Advanced Studies in Pure Mathematics, Vol. 10, Algebraic Geometry, Sendai, 1987, pp. 283-360.

[19] J. Kollár, T. Matsusaka, Riemann-Roch type inequalities, Amer. J. Math. 105, $1983,229-252$.

[20] J. Le Potier, Dualité etrange sur le plan projectif, Luminy 12-1996.

[21] J. Le Potier, Dualité etrange, sur le surfaces, preprint, Preliminary version 09-06-2003.

[22] A. Markman, Brill-Noether duality for moduli spaces of sheaves on K3 surfaces, J. Algebraic Geometry 10, 2002, pp. 623-694.

[23] A. Mayer, Families of K3 surfaces, Nagoya Math. J. 48, 1972, pp. 1-17.

[24] M. Maruyama, Moduli of stable sheaves, II, J. Math. Kyoto Univ. 18, 1978, pp. 557-614.

[25] C. T. McMullen, Dynamics on K3 surfaces: Salem numbers and Siegel disks, J. Reine Angew. Math. 545, 2002, pp. 201-233.

[26] S. Mukai, On the moduli space of bundles on K3 surfaces, I, Vector Bundles on Algebraic Varieties, TIFR, Bombay, O.U.P., 1987, pp. 341-413. 
[27] S. Mukai, Symplectic structure of the moduli space of sheaves on an abelian or K3 surface, Invent. math 77, 1984, pp. 101-116.

[28] S. Mukai, Curves, K3 surfaces and Fano 3-folds of genus $\leq 10$, Algebraic Geometry and Commutative Algebra, Vol. I, Kinokuniya Tokyo, 1988, pp. 357377.

[29] S. Mukai, Moduli of vector bundles on K3 surfaces and symplectic manifolds, Sugaku Expos. 1, 1988, pp. 139-174.

[30] K. O'Grady, The weight-two Hodge structure of moduli spaces of sheaves on a K3 surface, J. of Algebraic Geom. 6, 1997, pp. 599-644.

[31] J. H. Silverman, Rational points on K3 surfaces: a new canonical height, Invent. Math. 105, 1991, pp. 347-373.

[32] C. Voisin, Théoréme de Torelli pour les cubiques de $P^{5}$, Invent. Math. 86, 1986, pp. 577-601.

[33] C. Voisin, Sur la stabilité des sous-variétés lagrangiennes des variétés symplectiques holomorphes, Complex projective geometry (Trieste, 1989/Bergen, 1989), London Math. Soc. Lecture Note Ser. 179, Cambridge Univ. Press, Cambridge, 1992, pp. 294-303.

[34] C. Voisin, Théorie de Hodge et géometrie algébrique complexe, Cours spécialisés, Collection SMF 10, 2002.

[35] K. Yoshioka, Some examples of Mukai's reflections on K3 surfaces, J. reine angew. Math. 515, 1999, pp. 97-123.

[36] K. Yoshioka, Moduli spaces of stable sheaves on abelian surfaces, Math. Ann. 321, 2001, 817-884.

Kieran G. O'Grady

Università di Roma "La Sapienza",

Dipartimento di Matematica "Guido Castelnuovo",

Piazzale Aldo Moro n. 5, 00185 Rome, Italy,

e-mail: ogrady@mat.uniroma1.it. 